\title{
Notes on $\pi_{1}$ of Smooth Loci of log del Pezzo Surfaces
}

\author{
ChENYANG XU
}

\section{Introduction}

A projective surface $R$ over $\mathbb{C}$ is called a log del Pezzo surface if it contains only quotient singularities and if the canonical divisor $K_{R}$ is an anti-ample $\mathbb{Q}$-divisor. Although the fundamental group of $R$ is always trivial, the fundamental group of the smooth locus $\pi_{1}\left(R^{\mathrm{sm}}\right)$ is, in general, not zero. Nevertheless, it is known that such a group is always finite (cf. [GZ; KMc]). The aim of this paper is to determine these groups.

Our approach to this problem is as follows. Given a log del Pezzo surface $R$, we take the universal cover of its smooth locus $R^{\mathrm{sm}}$. Given that $\pi_{1}\left(R^{\mathrm{sm}}\right)$ is finite [GZ; KMc], the Riemann existence theorem (see [SGA1]) states that the universal cover is actually an algebraic variety. Therefore, we can take the normal closure $S$ of $R$ in the function field of this covering space. In this way we obtain a pair $\left(S, \pi_{1}\left(R^{\mathrm{sm}}\right)\right)$, where $S$ is also a log del Pezzo surface and $\pi_{1}\left(R^{\mathrm{sm}}\right)$ is a finite group acting on it, such that for every nontrivial element $g \in \pi_{1}\left(R^{\mathrm{sm}}\right)$ the fixed locus $S^{g}$ is isolated. We can also equivariantly resolve $S$ to get a smooth rational surface carrying the same finite group action. This motivates the following definitions.

1.1. Definition. We call a finite group $G$ acting on a normal surface $S$ an action with isolated fixed points (IFP) if $S$ has at worst quotient singularities and if, for every nonunit element $g \in G$, the fixed locus $S^{g}$ consists of finite points. Similarly, we call $(S, G)$ birational to an action with IFP if there is a $G$-equivariant birational proper model $S^{\prime}$ of $S$ such that $\left(S^{\prime}, G\right)$ is an action with IFP.

Now we can divide our question into three parts:

(1) finding all the birational classes $(S, G)$ that contain a representative $(\tilde{S}, G)$ with IFP;

(2) determining those groups $G$ for which we can choose $(\tilde{S}, G)$ as in (1) with the additional property that $K_{\tilde{S}}$ is anti-ample; and

(3) for any $G$ appearing in part (2), checking for the existence of $(\tilde{S}, G)$ satisfying $\pi_{1}\left(\tilde{S}^{\mathrm{sm}}\right)=e$.

All finite subgroups of the Cremona group are classified in [DI]. Based on their table, we can solve the part (1) of our question.

Received February 11, 2008. Revision received January 21, 2009. 
1.2. THEOREM. Let $G$ be a finite group acting on a rational surface $S$ such that $(S, G)$ is birational to an action with IFP. Then $G$ is precisely one of the following groups:

(i) a finite subgroup $G$ of $\mathrm{GL}_{2}(\mathbb{C})$ whose abelian subgroups are all cyclic;

(ii) a finite subgroup $G$ of $\mathrm{PGL}_{2}(\mathbb{C}) \times \mathrm{PGL}_{2}(\mathbb{C})$ whose subgroups as $G_{1} \times G_{2}$ have the property that $\left|G_{1}\right|$ and $\left|G_{2}\right|$ are coprime;

(iii) $\mathbb{Z} / n: \mathbb{Z} / 3$ or $\mathbb{Z} / 2 \times(\mathbb{Z} / n: \mathbb{Z} / 3)$, where $n$ is an odd integer and $\mathbb{Z} / n: \mathbb{Z} / 3$ means the group generated by $u:\left(x_{0}, x_{1}, x_{2}\right) \rightarrow\left(x_{1}, x_{2}, x_{0}\right)$ and $v:\left(x_{0}, x_{1}, x_{2}\right) \rightarrow\left(\varepsilon_{n} x_{0}, \varepsilon_{n}^{s} x_{1}, x_{2}\right)\left(s^{2}-s+1 \equiv 0 \bmod n\right)$, where $\varepsilon_{n}$ is a primitive $n$th root;

(iv) $F_{4 n}, G_{4 n}$, and $H_{4 n}$, which are groups of order $4 n$ (for the definition, see Proposition 3.9); or

(v) $(\mathbb{Z} / 3)^{2}: \mathbb{Z} / 2,(\mathbb{Z} / 3)^{2}: \mathbb{Z} / 4$, and $(\mathbb{Z} / 3)^{2}: Q_{8}$.

Although Theorem 1.2 is the strongest statement, we emphasize that there is a more conceptual version as follows.

1.3. THEOREM. A finite group $G$ acting on a smooth projective rational surface $S$ is an action birationally with IFP if and only if it satisfies the following conditions:

(i) for any point $x \in S$, every abelian subgroup of the stabilizer $G_{x}$ is cyclic; and

(ii) for any nonunit element $g \in G$, every curve $C \subset S^{g}$ satisfies genus $g(C)=0$.

In fact, in our case-by-case study the phenomenon can be formulated as a dichotomy as follows:

- for a simple rational surface (e.g., a Hirzebruch surface or a del Pezzo surface of degree $\geq 5$ ), the minimal action is birationally with IFP if and only if, for any point $x$, the stabilizer $G_{x}$ does not contain a noncyclic abelian subgroup;

- for other complicated rational surfaces, any minimal action contains a nontrivial element that fixes a positive genus curve pointwise.

In [Ko], a similar method is used to study the case when $G$ is abelian, yielding a list of possible first homology groups of log del Pezzo surfaces. By listing the abelian groups appearing in Theorem 1.2, we can refine the results there (cf. [Ko, 11]).

1.4. Corollary. Let $S$ be a log del Pezzo surface. Then $H_{1}\left(S^{\mathrm{sm}}, \mathbb{Z}\right)$ is one of the following groups: $(\mathbb{Z} / 3)^{2}, Z / 3 \times \mathbb{Z} / 6, \mathbb{Z} / 2 \times \mathbb{Z} / n$ ( $n$ is 4 or $\left.4 k+2\right)$, or $\mathbb{Z} / m$ for any $m$.

Then we can apply the equivariant minimal model program to answer part (2) of our question as follows.

1.5. Theorem. Suppose we can choose $(\tilde{S}, G)$ in Theorem 1.2 to satisfy the additional property that $\tilde{S}$ is a log del Pezzo surface. Then $G$ is precisely one of the groups listed in (i)-(iv) of that theorem.

Our aim in Section 5 of this paper is to answer part (3) of our question. We construct models $(\tilde{S}, G)$ that satisfy the property $\pi_{1}\left(\tilde{S}^{\mathrm{sm}}\right)=e$ for most groups $G$ in Theorem 1.2. Unfortunately, we leave three series of groups undetermined. 
ACKNOWLEDGMENTS. I am indebted to my advisor, János Kollár, for suggesting this question to me and also for his useful comments and crucial suggestions. Without his encouragement, this paper would never have been written. I thank Igor Dolgachev for sending me the new version of [DI]; as mentioned previously, our work relies on it substantially. I also thank Ruochuan Liu, Garving Luli, Yi Ni, and Zhiwei Yun for helpful conversations and also the referee for several suggestions.

\section{Local and Global Results}

See $[\mathrm{Br}]$ for a table of the quotient surface singularities, and observe that any quotient singularity is rational. In particular, given a resolution, the irreducible components of the exceptional locus are smooth rational curves and the configuration of the exceptional locus is a tree.

Now let $S$ be a projective rational surface with $G \subset \operatorname{Aut}(X)$ a finite group of automorphisms. In this section we aim to give some criteria for deciding whether $(S, G)$ is birational to an action with IFP. By abuse of notation, we use $\sum_{S, G}$ to denote both the set of the irreducible curves that are fixed pointwise by some nontrivial elements of $G$ and the corresponding reduced divisor. When the surface and the group are clear from the context, we will omit the subscript. We call a subset $\left\{C_{1}, C_{2}, \ldots, C_{n}\right\} \subset \sum_{S, G}$ a cycle if the intersection of $C_{i}$ and $C_{i+1}$ is nonempty for all $1 \leq i \leq n\left(C_{n+1}=C_{1}\right)$. We also use the same letter to denote both the divisor and its birational transforms on any other birational models. Define a subset

$$
\tilde{\Sigma}=\left\{C \in \sum \mid C \text { intersects with other curves of } \sum \text { in at most two points }\right\} .
$$

Let $x$ be a point in the smooth $G$-surface $S$ with the stabilizer $G_{x} \subset G$. In an analytical neighborhood of $x, S$ is isomorphic to a germ $\left(\mathbb{C}^{2}, 0\right)$ with a finite-group $G_{x} \subset \mathrm{GL}_{2}$ action. For the following lemma, we assume this identification.

2.1. LEMMA. If $G_{x}$ contains a noncyclic abelian group, then there are two curves $C_{1}, C_{2} \in \sum$ such that, if we denote by $\left\langle g_{1}\right\rangle$ (resp. $\left\langle g_{2}\right\rangle$ ) the subgroup fixing $C_{1}$ (resp. $\left.C_{2}\right)$ pointwise, then $\left\langle g_{1}, g_{2}\right\rangle \subset G_{x}$ gives a noncyclic abelian group. Furthermore, for any sequence of $G_{x}$-equivariant blow-ups $\pi: S \rightarrow \mathbb{C}^{2}, C_{1}$ and $C_{2}$ belong to the same connected component of $\sum_{S}$.

Proof. Since $G_{x}$ contains a noncyclic abelian group, we know that $(\mathbb{Z} / p)^{2} \subset G_{x}$ for some prime $p$. Given a $G$-surface $S$, we have $\sum_{(\mathbb{Z} / p)^{2}} \subset \sum_{G}$; hence it suffices to prove the claim for $G_{x} \cong(\mathbb{Z} / p)^{2}$. But if we choose the basis suitably then any embedding of $(\mathbb{Z} / p)^{2}$ to $\mathrm{GL}_{2}(\mathbb{C})$ is conjugate to the embedding $(0,1) \rightarrow$ $\operatorname{diag}\left\{1, e^{2 \pi i / p}\right\}$ and $(1,0) \rightarrow \operatorname{diag}\left\{e^{2 \pi i / p}, 1\right\}$, so the first statement is obvious.

To prove the second statement, we can just look at the blow-ups whose centers are the intersection points of (at least) two curves in $\sum_{S}$. Assume after blowing up $x$ that $\pi: S_{1} \rightarrow \mathbb{C}^{2}$ with the exceptional divisor $E=\pi^{-1}(0)$. The action can be lifted from $\mathbb{C}^{2}$ to $S_{1}$ such that $G_{x}$ acts on $E$ through the natural homomorphism $p: \mathrm{GL}_{2}(\mathbb{C}) \rightarrow \mathrm{PGL}_{2}(\mathbb{C})$. The restriction of $p$ on $(\mathbb{Z} / p)^{2}$ has a nontrivial kernel that fixes $E$ pointwise.

Furthermore, $(\mathbb{Z} / p)^{2}$ fixes the two intersection points of $C_{i}(i=1,2)$ and $E$, so if we replace $C_{1}$ and $C_{2}$ by $C_{i}$ and $E$ then the lemma's assumptions still hold. 
Repeating the argument, we can see that there exists no sequence of equivariant blow-ups $\pi: S \rightarrow \mathbb{C}^{2}$ that separates the birational transforms of $C_{1}$ and $C_{2}$ into different connected components of $\sum_{S}$.

This local computation leads to the following global version.

2.2. Corollary. Let a finite group $G$ act on a smooth surface S. Assume that $\sum_{S}$ contains a cycle $\left\{C_{1}, C_{2}, \ldots, C_{n}\right\}$ and that $\left\langle g_{i}\right\rangle$ fixes $C_{i}$ pointwise. If $\left\langle g_{i}, g_{i+1}\right\rangle \subset G$ is noncyclic abelian for every $i$, then the action is not birational to one with only IFP.

We can also prove the converse of Lemma 2.1 as follows.

2.3. LemMA. With notation as in Lemma 2.1, if $G_{x}$ is cyclic and if $C_{1}, C_{2}$ are the curves fixed by some nontivial subgroups of $G_{x}$ (there are at most two such curves), then there is a sequence of $G_{x}$-equivariant blow-ups $\pi: S \rightarrow \mathbb{C}^{2}$ such that the strict transforms of $C_{1}, C_{2}$ are disconnected in the configuration of $\sum_{S}$. Given a $C_{i}$, we can assume that all exceptional curves $E_{i} \subset \sum_{S}$ form a tail attaching to it.

Proof. We assume the order- $r$ cyclic group $G_{x}$ is generated by $\left(e^{2 p \pi i / r}, e^{2 q \pi i / r}\right)$, where $(p, q)=1$. Blowing up $x$, we will change an intersection point from type $\frac{1}{r}(p, q)$ to two intersection points of types $\frac{1}{r}(p, q-p)$ and $\frac{1}{r}(p-q, q)$. If we continue blowing up the new intersection points and changing the action in this way, we can choose $t$ with $(r,(t+1) p-q)=1$ so that the image of $\frac{1}{r}(p, q-t p)$ in $\mathrm{PGL}_{2}(\mathbb{C})$ is of order $r$. This means that the unit is the only element of $G_{x}$ that fixes the corresponding exceptional curve.

The global version of Lemma 2.3 is as follows.

2.4. LEMMA. Suppose a finite group $G$ acts on a smooth rational surface $S$ with the following properties:

(i) for any nonunit element, its fixed locus consists only of smooth rational curves and isolated points;

(ii) every intersection point of two curves in $\sum_{S}$ has an abelian stabilizer; and

(iii) after separating the pair of irreducible curves contained in $\tilde{\sum}$ that intersect at a point with cyclic stabilizer, every component of the configuration of $\sum_{S}$ is a chain.

Then $(S, G)$ is birational to an action with IFP.

Proof. For every point $x$ satisfying condition (iii), by Lemma 2.3 we know there exists a sequence of $G$-equivariant blow-ups $S^{\prime} \rightarrow S$ that separates the two branches in $\sum_{S}$ containing $x$ and then adds a tail only to the component in $\tilde{\sum}$. Hence, the configuration of $\sum_{S^{\prime}}$ is a disjoint union of chains.

In order to contract $\sum_{S^{\prime}}$, we need the self-intersection of each component in $\sum_{S^{\prime}}$ to be less than or equal to -2 . This may not be true for $S^{\prime}$. However, we can blow up general orbits on curves in $\sum_{S^{\prime}}$. The exceptional locus created in this way 
satisfies the property that, for any nontrivial element $g \in G$ acting on it, the fixed locus is isolated. Hence, after a sequence of such blow-ups, we can assume (a) that there is a surface $S^{\prime \prime}$ with a proper $G$-equivariant birational morphism $f^{\prime}: S^{\prime \prime} \rightarrow S^{\prime}$ inducing an isomorphism from $\sum_{S^{\prime \prime}}$ to $\sum_{S^{\prime}}$ and (b) that all the irreducible components in $\sum_{S^{\prime \prime}}$ have self-intersection numbers less than or equal to -2 . From the table of quotient surface singularities in [Br], we can contract every connected component of $\sum_{S^{\prime \prime}}$ (which is a chain) to a cyclic quotient surface singularity.

2.5. Lemma. For any $(S, G)$, there exists an equivariant blow-up $\pi: S^{\prime} \rightarrow S$ such that Lemma 2.4(ii) holds for $S^{\prime}$.

Proof. Assume we have a point $x \in S$ with a nonabelian stabilizer $G_{x} \in \mathrm{GL}_{2}(C)$. Blowing up $x$ yields an exceptional divisor $E$. Then, for any point $y \in E$, the stabilizer $G_{y}$ fits the exact sequence

$$
1 \rightarrow K \rightarrow G_{y} \rightarrow H \rightarrow 1,
$$

where $K$ is the kernel of $\rho: G_{x} \rightarrow \mathrm{PGL}_{2}(\mathbb{C})$ and $H$ is the stabilizer of $y$ for the induced action $\operatorname{im}(\rho)$ on $E$. Since $H$ is abelian and since $K$ is in the center of $\mathrm{GL}_{2}(\mathbb{C})$, we conclude that $G_{y}$ is an abelian group.

Although Corollary 2.2 and Lemma 2.4 do not summarize all possible cases, they are sufficient for our purpose when combined with the following simple lemma.

2.6. LEMMA. Let $g$ be a nontrivial element in $G$. If there is a curve $C \in S^{g}$ with genus $g(C)>0$, then $(S, G)$ is not birational to an action with IFP.

Proof. For any birational $G$-map $f: S \rightarrow S^{\prime}$, if $S^{\prime}$ has only quotient singularities then $f$ cannot contract $C$, so $\left(S^{\prime}, G\right)$ cannot be with only IFP.

\section{Birational Classification of Actions on Rational Surfaces with Isolated Fixed Points}

The aim of this section is to give a complete classification of actions on a rational surface $(S, G)$ that have birational $G$-models with only IFP. For a given surface $S$, the actions are classified up to the conjugation of the automorphism of $S$. A priori, having such a model is a birational property, so we will consider only the minimal actions; for example, if there is a birational $G$-morphism $f: S \rightarrow S^{\prime}$ then it is, in fact, an isomorphism.

3.1. Notation. We employ the following standard notation for groups:

- $\mathbb{Z} / n$ denotes the order- $n$ cyclic group;

- $S_{n}$, the permutation group of degree $n$;

- $A_{n}$, the alternating group of degree $n$;

- $D_{2 n}$, the dihedral group of order $2 n$;

- $Q_{4 n}=\left\langle a, b \mid a_{2 n}=1, b_{2}=a_{n}, b^{-1} a b=a^{-1}\right\rangle$, a dicyclic group of order $4 n$, is a generalized quaternion group if $n=2 k$; 
- $L_{n}(q)=\operatorname{PSL}\left(n, \mathbb{F}_{q}\right)$, where $q=p^{r}$ is a power of a prime number $p$;

- $H_{n}(p)$, the Heisenberg group of unipotent $n \times n$ matrices with entries in $\mathbb{F}_{p}$;

- $A \bullet B$ is an upward extension of $B$ with the aid of a normal subgroup $A$;

- $A: B$ is a split extension (i.e., a semi-direct product $A \rtimes B$ defined by a homomorphism $\varphi: B \rightarrow \operatorname{Aut}(A))$;

- $A$ 2 $S_{n}$ is the wreath product, so $A^{n}: S_{n}$ and $S_{n}$ acts on $A^{n}$ by permuting the factors;

- $\left(G_{1}, H_{1}, G_{2}, H_{2}\right)_{\alpha}$ denotes the subgroup of $G_{1} \times G_{2}$ consisting of elements $\left\{\left(g_{1}, g_{2}\right) \mid g_{1}\right.$ and $g_{2}$ have the same image under the isomorphism $\alpha: G_{1} / H_{1} \cong$ $\left.G_{2} / H_{2}\right\}$ (we omit the $\alpha$ if the isomorphism is clear);

- $\mu_{n}$, the group of $n$th roots of unity with generator $\varepsilon_{n}=e^{2 \pi i / n}$.

We also require notation for polyhedron groups, which are precisely all the possible finite subgroups of $\mathrm{PGL}_{2}$ :

- a cyclic group $\mathbb{Z} / n$ of order $n$;

- a dihedral group $D_{2} n$ of order $2 n$;

- the tetrahedral group $T \cong A_{4}$ of order 12 ;

- the octahedral group $O \cong S_{4}$ of order 24;

- the icosahedral group $I \cong A_{5}$ of order 60 .

We will use $\bar{T}, \bar{O}$, and $\bar{I}$ to denote the respective double covers of $T, O$, and $I$ under the homomorphism

$$
\mathrm{SL}_{2} \rightarrow \mathrm{PGL}_{2} \text {. }
$$

Note that the double cover of $D_{2 n}$ is $Q_{4 n}$.

3.2. Remark. For the discussions that follow in this section, we rely heavily on the results in [DI]. In fact, we will do a case-by-case study for Sections 4-6 of their paper.

$$
\text { The Case } S=\mathbb{P}^{2}
$$

We recall some standard terminology from the theory of linear groups. Let $G$ be a finite subgroup of the general linear group GL $(V)$ of a complex vector space $V$. The group $G$ is called intransitive if the representation of $G$ in $V$ is reducible; otherwise, it is called transitive. A transitive group $G$ is called imprimitive if it contains an intransitive normal subgroup $G^{\prime}$. In this case, $V$ decomposes into a direct sum of $G^{\prime}$-invariant proper subspaces and the elements from $G$ permute them. A group is primitive if it is neither intransitive nor imprimitive. We reserve this terminology for subgroups of PGL $(V)$ while keeping in mind that each such group can be represented by a subgroup of GL( $V)$.

Since any element $g$ of finite order in $\mathrm{PGL}_{3}(\mathbb{C})$ can be lifted as an element of $\mathrm{GL}_{3}(\mathbb{C})$, it follows that $g$ fixes a curve pointwise if and only if the characteristic polynomial of the lifting has multiple roots-in which case the curve is a line.

\section{Intransitive Actions}

For any intransitve group action $\left(\mathbb{P}^{2}, G\right)$, we see that $G$ also acts linearly on $\mathbb{C}^{2}$ with an equivariant embedding $i: \mathbb{C}^{2} \rightarrow \mathbb{P}^{2}$; hence $G \subset \mathrm{GL}_{2}$. 
3.3. Proposition. An intransitive action $G$ on $\mathbb{P}^{2}$ is birational to an action with IFP if and only if any abelian subgroup $H \subset G$ is cyclic.

Proof. Blowing up the origin of $\mathbb{C}^{2}$, we know that if any abelian subgroup of $G$ is cyclic then all the conditions of Lemma 2.4 hold for this ruled surface. To prove the "only if" part, we observe that if $G$ has a noncyclic abelian subgroup then the cycle in $\sum_{S}$ consisting of $\left\{x_{0}=0, x_{1}=0, x_{2}=0\right\}$ satisfies the assumptions of Corollary 2.2. Therefore, $\left(\mathbb{P}^{2}, G\right)$ is not birational to any action with IFP.

Dolgachev and Iskovskikh classify all such finite $G$ [DI, Lemma 4.6]. To find all transitive actions that are birational to the ones with IFP, we need to find all $G$ that do not contain any noncyclic subgroup. They are listed below, which gives a subtable of [DI, Lemma 4.5 and Form 4.1]. Here we use $\tilde{G}$ to denote the preimage of $G$ in $\mathbb{C}^{*} \times \mathrm{SL}_{2}(\mathbb{C})$.

(1) $G \cong(\mathbb{Z} / m k, \mathbb{Z} / m, \mathbb{Z} / n k, \mathbb{Z} / n)_{\alpha}(\alpha$ is an automorphism of $\mathbb{Z} / k)$, where $\operatorname{gcd}(m, n)=1$

(2) $\tilde{G} \cong\left(\mu_{2 m}, \mu_{2 m}, H, H\right)$ and $G \cong \mu_{m} \times H$, where $H$ is a nonabelian binary polyhedral group and $\operatorname{gcd}(m,|H|)=1$;

(3) $\tilde{G} \cong\left(\mu_{6 m}, \mu_{2 m}, \bar{T}, Q_{8}\right)$ and $G \cong\left(\mu_{m} \times Q_{8}\right) . \mathbb{Z} / 3$, where $\operatorname{gcd}(m, 2)=1$;

(4) $\tilde{G} \cong\left(\mu_{4 m}, \mu_{2 m}, Q_{4 n}, \mathbb{Z} / n\right)$ and $G \cong\left(\mu_{2 m} \times \mu_{n}\right) \cdot \mathbb{Z} / 2$, where $m$ is even and $\operatorname{gcd}(m, n)=1$

(5) $\tilde{G} \cong\left(\mu_{4 m}, \mu_{m}, Q_{4 n}, \mathbb{Z} / n\right)$ and $G \cong \mu_{m} \times D_{2 n}$, where $n$ is odd and $\operatorname{gcd}(m, n)=1$

(6) $\tilde{G} \cong\left(\mu_{4 m}, \mu_{2 m}, Q_{8 n}, Q_{4 n}\right)$ and $G \cong\left(\mu_{m} \times Q_{4 n}\right) .2$, where $\operatorname{gcd}(m, 2 n)=1$.

\section{Transitive Imprimitive Actions}

3.4. LEMMA [DI, Thm. 4.7]. Let $G$ be a transitive imprimitive finite subgroup of $\mathrm{PGL}_{3}$. Then $G$ is conjugate to one of the following groups:

- $G \cong(\mathbb{Z} / n)^{2}: Z / 3$ generated by transformations

$$
\left[\varepsilon_{n} x_{0}, x_{1}, x_{2}\right],\left[x_{0}, \varepsilon_{n} x_{1}, x_{2}\right],\left[x_{2}, x_{0}, x_{1}\right]
$$

- $G \cong(\mathbb{Z} / n)^{2}: S_{3}$ generated by transformations

$$
\left[\varepsilon_{n} x_{0}, x_{1}, x_{2}\right],\left[x_{0}, \varepsilon_{n} x_{1}, x_{2}\right],\left[x_{0}, x_{2}, x_{1}\right],\left[x_{2}, x_{0}, x_{1}\right]
$$

- $G=G_{n, k, s} \cong\left(\mathbb{Z} / n \times \mathbb{Z} / \frac{n}{k}\right): \mathbb{Z} / 3$, where $k>1, k \mid n$, and $s^{2}-s+1=0$ modulo $k$. It is generated by transformations

$$
\left[\varepsilon_{n / k} x_{0}, x_{1}, x_{2}\right],\left[\varepsilon_{n}^{s} x_{0}, \varepsilon_{n} x_{1}, x_{2}\right],\left[x_{2}, x_{0}, x_{1}\right]
$$

- $G \cong\left(\mathbb{Z} / n \times \mathbb{Z} / \frac{n}{3}\right): S_{3}$ generated by transformations

$$
\left[\varepsilon_{n / 3} x_{0}, x_{1}, x_{2}\right],\left[\varepsilon_{n}^{2} x_{0}, \varepsilon_{n} x_{1}, x_{2}\right],\left[x_{0}, x_{2}, x_{1}\right],\left[x_{1}, x_{0}, x_{2}\right] .
$$

3.5. EXAmple. $\left.G=(\mathbb{Z} / 3)^{2}: \mathbb{Z} / 2\right)$ is generated by

$$
\left[\varepsilon_{3}^{2} x_{0}, \varepsilon_{3} x_{1}, x_{2}\right],\left[x_{2}, x_{0}, x_{1}\right],\left[x_{0}, x_{2}, x_{1}\right] \text {. }
$$


$\sum_{\mathbb{P}^{2}}$ contains nine lines $\left\{x_{i}=\varepsilon_{3}^{k} x_{j}\right\}$, which is the Hessian arrangement: each line passes through exactly four points of $\left\{\left(1, \varepsilon_{3}^{i}, \varepsilon_{3}^{j}\right),(1,0,0),(0,1,0),(0,0,1)\right\}$, where $0 \leq i, j \leq 2$. Through each of these twelve points, there are exactly three lines in $\sum_{\mathbb{P}^{2}}$. Blowing up the twelve points and then contracting the birational transform of $\sum_{\mathbb{P}^{2}}$, we get an action with IFP and $3 K_{S}$ is a trivial Cartier divisor.

3.6. Proposition. All transitive imprimitive actions $\left(\mathbb{P}^{2}, G\right)$ that are birational to the ones with IFP have $G$ as one of the following three groups: $S_{3}, \mathbb{Z} / 3: S_{3} \cong$ $(\mathbb{Z} / 3)^{2}: 2$, or $\mathbb{Z} / n: \mathbb{Z} / 3$.

Proof. When $G=(\mathbb{Z} / n)^{2}: K\left(K=\mathbb{Z} / 3\right.$ or $\left.S_{3}\right)$ and $n>1$, the subgroup action $\left(\mathbb{P}^{2},(\mathbb{Z} / n)^{2}\right)$ is not birational to an action with only IFP. In fact, the cycle $\left\{x_{0}=0, x_{1}=0, x_{2}=0\right\} \subset \sum_{\mathbb{P}^{2}}$ satisfies the assumptions of Corollary 2.2. When $n=1$, after possibly blowing up $(1,1,1)$ we can see that the group action satisfies all the conditions of Lemma 2.4.

A similar argument shows that if $G=G_{n, k, s}: \mathbb{Z} / 3$ then the action is not birational to an action with IFP when $k \neq n$. For $\mathbb{Z} / n: \mathbb{Z} / 3$, it acts on $\mathbb{P}^{2}$ with IFP.

For the last case, we need only consider when $n=3$. Since for this case $G=$ $\mathbb{Z} / 3: S_{3}$, we obtain an equivariant birational model with IFP as in Example 3.5.

\section{Primitive Actions}

For the classical cases of finite primitive actions on $\mathbb{P}^{2}$ (a table of all such actions is given in [B] or [DI, Thm. 4.8]), we have the following result.

3.7. Proposition. (i) The action of the icosahedron group $A_{5}$ on $\mathbb{P}_{2}$ that leaves a nonsingular conic invariant is not birational to any action with IFP.

(ii) The action of the Hessian group Hes $\cong(\mathbb{Z} / 3)^{2}: \bar{T}$ that is the automorphism group of the Hessian pencil

$$
x^{3}+y^{3}+z^{3}+t x y z=0
$$

is not birational to any action with IFP.

(iii) The actions of the subgroups $G$ of the Hessian group, where $G=(\mathbb{Z} / 3)^{2}$ : $(\mathbb{Z} / 4)$ and $(\mathbb{Z} / 3)^{2}: Q_{8}$, are birational to actions with IFP.

(iv) The action of the Klein group $L_{2}(7)$ of order 168, which is the automorphism group of the Klein quartic

$$
x^{3} y+y^{3} z+z^{3} x=0
$$

is not birational to any action with IFP.

(v) The action of the Valentiner group of order $360\left(\cong A_{6}\right)$, which can be realized as the full group of automorphisms of the nonsingular plane sextic

$$
10 x^{3} y^{3}+9 z x^{5}+9 z y^{5}-45 x^{2} y^{2} z^{2}-135 x y z^{4}+27 z^{6}=0,
$$

is not birational to any action with IFP.

Proof. We check the claim case by case.

(i) Since $A_{5}$ acts on $\mathbb{P}^{1}$, it acts on the complete linear system of $\mathcal{O}(2)$, which is isomorphic to $\mathbb{P}^{2}$. Choose $\left(x_{0}^{2}, x_{0} x_{1}, x_{1}^{2}\right)$ to be the basis of $\mathbb{P}^{2}$. Now $A_{5}$ has 
a subgroup $D_{4}$ whose nontrivial elements act on $\mathbb{P}^{1}$ as $\left(x_{0}, x_{1}\right) \rightarrow\left(x_{1}, x_{0}\right)$, $\left(x_{0}, x_{1}\right) \rightarrow\left(x_{1},-x_{0}\right)$, and $\left(x_{0}, x_{1}\right) \rightarrow\left(-x_{0}, x_{1}\right)$. Then the respective induced actions on $\mathbb{P}^{2}$ fix three lines $\left(y_{0}-y_{2}=0\right),\left(y_{0}+y_{2}=0\right)$, and $\left(y_{1}=0\right)$. By Corollary 2.2, we see that this action of $A_{5}$ is not birational to one with IFP.

(ii) The Hessian group $G_{216}$ has a homomorphism to $\mathrm{PSL}_{2}$ that induces the following exact sequence

$$
1 \rightarrow \mathbb{Z}_{3}: S_{3} \rightarrow G_{216} \rightarrow A_{4} \rightarrow 1 \text {. }
$$

We can write a generator of $G_{216}$ as follows. The kernel $\mathbb{Z} / 3: S_{3}$ is the group as in Example 3.5, and we have another three generators (cf. [D, 3.1.4]):

$$
\sigma_{1}=\left(\begin{array}{ccc}
1 & 1 & 1 \\
1 & \varepsilon & \varepsilon^{2} \\
1 & \varepsilon^{2} & \varepsilon
\end{array}\right), \quad \sigma_{2}=\left(\begin{array}{ccc}
1 & \varepsilon & \varepsilon \\
\varepsilon^{2} & \varepsilon & \varepsilon^{2} \\
\varepsilon^{2} & \varepsilon^{2} & \varepsilon
\end{array}\right), \quad \sigma_{3}=\left[\varepsilon_{3} x_{0}, x_{2}, x_{1}\right] .
$$

We observe that there is a subgroup of the Hessian group generated by

$$
u:\left(x_{0}, x_{1}, x_{2}\right) \rightarrow\left(\omega x_{0}, x_{1}, x_{2}\right) \text { and } v:\left(x_{0}, x_{1}, x_{2}\right) \rightarrow\left(x_{0}, \omega x_{1}, x_{2}\right) .
$$

By Proposition 3.6, we know that this $(\mathbb{Z} / 3)^{2}$ action is not birational to an action with IFP.

(iii) In the exact sequence just displayed in paragraph (ii), $A_{4}$ has a subgroup $\mathbb{Z} / 2$ that is generated by the image of $\sigma_{1}$ and a subgroup $(\mathbb{Z} / 2)^{2}$ that is generated by the image of $\sigma_{1}$ and $\sigma_{2}$. Because $(\mathbb{Z} / 3)^{2}:(\mathbb{Z} / 4)$ is a subgroup of $(\mathbb{Z} / 3)^{2}: Q_{8}$, we only have to prove the statement for the second case. Now the subgroup $(\mathbb{Z} / 3)^{2}$ generated by $\left(x_{0}, x_{1}, x_{2}\right) \rightarrow\left(\omega x_{0}, \omega^{2} x_{1}, x_{2}\right)$ and $\left(x_{0}, x_{1}, x_{2}\right) \rightarrow\left(x_{2}, x_{0}, x_{1}\right)$ is the only noncyclic abelian subgroup of $(\mathbb{Z} / 3)^{2}: Q_{8}$. In this case, we can easily check that $(\mathbb{Z} / 3)^{2}: Q_{8}$ acts on the model we construct in Example 3.5, which gives an action with IFP.

(iv) By [D, 6.5.2], the Klein quartic $x_{0}^{3} x_{1}+x_{1}^{3} x_{2}+x_{2}^{3} x_{0}=0$ is a specialization of the quartic

$$
C_{a, b, c}: x_{0}^{4}+x_{1}^{4}+x_{2}^{4}+a x_{0}^{2} x_{1}^{2}+b x_{1}^{2} x_{2}^{2}+c x_{0}^{2} x_{2}^{2}=0
$$

when $a=b=c=(-1+\sqrt{7}) / 2$. Notice that $(\mathbb{Z} / 2)^{2}$ acts on a general $C_{a, b, c}$ and that the induced action on $\mathbb{P}^{2}$ is not birational to any action with IFP. Thus, the Klein group action is not birational to any action with IFP.

(v) By [B], the Valentiner group will contain the icosahedron group as a subgroup; then, by part (i), we know it is not birational to any action with IFP.

$$
\text { The Case } S=\mathbb{P}^{1} \times \mathbb{P}^{1}
$$

Since $\operatorname{Aut}(S)=\mathrm{PGL}_{2} 2 S_{2}$, it follows that every finite group of $\operatorname{Aut}(S)$ has a subgroup $G^{0}$ in $\mathrm{PGL}_{2} \times \mathrm{PGL}_{2}$ with index at most 2 . Let $G^{0} \cap\left(\mathrm{PGL}_{2} \times\{e\}\right)=G_{1}$, $G^{0} \cap\left(\{e\} \times \mathrm{PGL}_{2}\right)=G_{1}$, and $H^{0}:=G_{1} \times G_{2}$.

3.8. Proposition. Let $G=G^{0}$. Then $\left(\mathbb{F}_{0}, G\right)$ is birational to an action with IFP if and only if $\left|G_{1}\right|$ and $\left|G_{2}\right|$ are coprime. 
Proof. If $G^{0}$ contains a subgroup of the form $G_{1} \times G_{2}$ and if the cardinalities of $G_{1}, G_{2}$ are not coprime, then $G^{0}$ contains a subgroup that is conjugate to $\mu_{n} \times \mu_{n}$. For $\mu_{n} \times \mu_{n}$ acting on $\mathbb{P}^{1} \times \mathbb{P}^{1}$, we observe that $\left\{x_{0}=0, x_{1}=0, y_{0}=0\right.$, $\left.y_{1}=0\right\}$ gives a cycle in $\sum_{\mathbb{P}^{1} \times \mathbb{P}^{1}, G}$ that satisfies the assumptions of Corollary 2.2. Conversely, it suffices to verify the three conditions of Lemma 2.4. Because a nonunit element $g \in G$ that fixes a curve must be in $G_{1}$ or $G_{2}$, the curves in $\sum$ are fibers of one of the projections. The assumption that $\left|G_{1}\right|$ and $\left|G_{2}\right|$ are coprime indeed implies that one of them, say $G_{1}$, is cyclic. Then there are at most two fibers of the form $p t \times \mathbb{P}^{1}$ in $\sum$, so a curve $C \in \sum$ of the form $\mathbb{P}^{1} \times p t$ must belong to $\tilde{\sum}$. We claim that any abelian subgroup of the stabilizer of a point is cyclic. In fact, such a stabilizer group will be isomorphic to a group of the form $(\mathbb{Z} / m k, \mathbb{Z} / m, \mathbb{Z} / n k, \mathbb{Z} / n)_{\alpha}$. Then the requirement of its abelian subgroups being cyclic is equivalent to $\operatorname{gcd}(m, n)=1$, which is equivalent to our coprimeness assumption. Hence, we can apply Lemma 2.4 to this case.

We list all possible actions in the case when $G=G^{0}$ :

(1) $G_{1} \times G_{2}$, where $\left|G_{1}\right|$ and $\left|G_{2}\right|$ are coprime;

(2) $(G, 1, G, 1)_{\alpha}=\left\{(g, \alpha(g)) \mid G=\mathbb{Z} / n, D_{2 n}, T\right.$, O, or $\left.I\right\}$;

(3) $\left(D_{2 m}, \mathbb{Z} / m, O, T\right), \operatorname{gcd}(m, 6)=1$;

(4) $\left(D_{2 m}, \mathbb{Z} / m, O,(\mathbb{Z} / 2)^{2}\right), \operatorname{gcd}(m, 2)=1$;

(5) $(\mathbb{Z} / 2 m, \mathbb{Z} / m, O, T), \operatorname{gcd}(m, 6)=1$;

(6) $\left(\mathbb{Z} / 3 m, \mathbb{Z} / m, T, D_{4}\right), \operatorname{gcd}(m, 2)=1$;

(7) $\left(D_{2 m}, \mathbb{Z} / m, D_{4 n}, D_{2 n}\right), \operatorname{gcd}(m, 2 n)=1$;

(8) $\left(\mathbb{Z}_{2 m}, \mathbb{Z} / m, D_{2 n}, \mathbb{Z}_{n}\right), \operatorname{gcd}(m, n)=1$;

(9) $\left(D_{2 m k}, \mathbb{Z} / m, D_{2 n k}, \mathbb{Z} / n\right)_{\alpha}, \operatorname{gcd}(m, n)=1$;

(10) $\left(\mathbb{Z} / 2 m, \mathbb{Z} / m, D_{2 n}, D_{n}\right), \operatorname{gcd}(m, 2 n)=1$;

(11) $(\mathbb{Z} / m k, \mathbb{Z} / m, \mathbb{Z} / n k, \mathbb{Z} / n)_{\alpha}, \operatorname{gcd}(m, n)=1$.

For the argument later, we point out that in case (9) the group $G$ is isomorphic to $D_{2 m n k}$ when $k>2$. When $k=2$ there are two groups: besides $D_{4 m n}$, there is another action that is birational to the action $\left(D_{2 m}, \mathbb{Z} / m, D_{4 n}, D_{2 n}\right)$ as in case (7).

3.9. Proposition. If $\left[G: G^{0}\right]=2$ and $\left(\mathbb{F}_{0}, G\right)$ is birational to an action with IFP, then $G$ is one of the following groups: $\mathbb{Z} / 2 n, D_{2 n}$ ( $n$ is odd or $4 k$ ), $F_{4 n}$ ( $n=$ $4 k+2)$, or $G_{4 n}(n=4 k+2)$. When $p^{2} \equiv-1 \bmod n$ has a solution, we also have $H_{4 n}, I_{4 n}$ ( $n$ even) and $J_{4 n}$ ( $n$ odd ), where:

(i) $F_{4 n}$ is the group generated by

$(x, y) \rightarrow\left(-\frac{1}{x},-\frac{1}{y}\right),(x, y) \rightarrow\left(e^{2 i \pi / n} x, e^{2 i \pi / n} y\right)$, and $(x, y) \rightarrow\left(e^{i \pi / n} y,-e^{i \pi / n} x\right) ;$

(ii) $G_{4 n}$ is the group generated by

$$
(x, y) \rightarrow\left(-\frac{1}{x},-\frac{1}{y}\right),(x, y) \rightarrow\left(e^{2 i \pi / n} x, e^{2 i \pi / n} y\right), \text { and }(x, y) \rightarrow(-y, x) ;
$$

(iii) $H_{4 n}$ is the group generated by

$$
(x, y) \rightarrow\left(-\frac{1}{x},-\frac{1}{y}\right),(x, y) \rightarrow\left(e^{2 i \pi / n} x, e^{2 p i \pi / n} y\right), \text { and }(x, y) \rightarrow\left(-\frac{1}{y}, x\right) ;
$$


(iv) $I_{4 n}$ is the group generated by

$$
(x, y) \rightarrow\left(-\frac{1}{x},-\frac{1}{y}\right),(x, y) \rightarrow\left(e^{2 i \pi / n} x, e^{2 p i \pi / n} y\right), \text { and }(x, y) \rightarrow\left(-\frac{1}{y},-x\right) ;
$$

(v) $J_{4 n}$ is the group generated by

$$
(x, y) \rightarrow\left(-\frac{1}{x},-\frac{1}{y}\right),(x, y) \rightarrow\left(e^{2 i \pi / n} x, e^{2 p i \pi / n} y\right), \text { and }(x, y) \rightarrow\left(\frac{1}{y},-x\right) .
$$

Proof. In general, $G^{0}$ has the form $(G, H, G, H)_{\alpha}$, where $G$ is given by the projection of $G^{0}$ on each factor. As in the argument of Proposition 3.8, we know that the only possible case is $H=1$; hence $G=G^{0} \bullet \mathbb{Z} / 2$, where $G^{0}$ is a polyhedral group. An element $h \in G$ whose image in $\mathbb{Z} / 2$ is nontrivial can be represented as $\left(h_{1}, h_{2}\right) \tau$, where $\tau$ is the element of switching two factors. We remark that $\left(h_{1}, h_{2}\right) \tau$ fixes a curve if and only if $h_{1}=h_{2}^{-1}$, which is also equivalent to saying that $\left(h_{1}, h_{2}\right) \tau$ has order 2 ; in this case, it fixes the curve $\left(h_{1}(y), y\right)$. So if

$$
G \text { contains a subgroup with the property } H \cong(\mathbb{Z} / 2)^{2}, H \subsetneq G^{0} \text {, }
$$

then we can apply Corollary 2.2 to show that $\left(\mathbb{F}_{0}, G\right)$ is not birational with IFP.

We claim that if $G$ acts on $\mathbb{F}_{0}$ birationally with IFP, then $G^{0}$ is either cyclic or dihedral. Since both $I$ and $O$ contain $T$, we need only rule out the case $G^{0}=T$. Because Aut $\left(A_{4}\right) \cong S_{4}$, we can assume (after taking a conjugation of an element in $\left.\mathrm{PGL}_{2}\right)$ that $\alpha=$ id. Now if $(g, g) \in G^{0}$ then $h^{-1}(g, g) h=\left(h_{2}^{-1} g h_{2}, h_{1}^{-1} g h_{1}\right) \in G^{0}$ and so $h_{1} h_{2}^{-1}$ is a commutator of $T$ in $\mathrm{PGL}_{2}$, which implies $h_{1}=h_{2}$. Furthermore, $h_{1}$ is in the normalizer. So $G$ is either $T \times \mathbb{Z} / 2\left(h_{1} \in T\right)$ or $O$. In either case, $G$ has a subgroup $H$ satisfying $(*)$.

Suppose $G^{0}=(\mathbb{Z} / n, 1, \mathbb{Z} / n, 1)_{s}$ with $\operatorname{gcd}(n, s)=1$. Then, since $G$ is an extension of $\mathbb{Z} / 2$ by $\mathbb{Z} / n$, we have $G=\mathbb{Z} / 2 n, \mathbb{Z} / n+\mathbb{Z} / 2$ ( $n$ even), or $D_{2 n}$; note that $\mathbb{Z} / n+\mathbb{Z} / 2$ ( $n$ even) and $D_{2 n}\left(n\right.$ even) both satisfy $(*)$. On the other hand, $\sum_{\mathbb{Z} / 2 n}$ is either empty or a single curve; and $\sum_{D_{2 n}}$ ( $n$ odd) consists of precisely $n$ rational curves, any two of which intersect at two indentical points. Blowing up these two points yields a model satisfying Lemma 2.4. Hence, we conclude that the action of $G$ on $\mathbb{F}_{0}$ is birational with IFP if and only if $G=\mathbb{Z} / 2 n$ or $G=D_{4 k+2}$.

Now suppose that $G^{0}=\left(D_{2 n}, 1, D_{2 n}, 1\right)_{\alpha}$ is generated by $a, b$ with $a^{n}=1$ and $b^{2}=1$. We will choose the representation $a(z)=e^{2 i \pi / n} z$ and $b(z)=-\frac{1}{z}$, after which we can assume that $\alpha(b)=b$ by composing $\alpha$ with an action of an element in $\mathrm{PGL}_{2}$. When $n=2$, it is easy to see that $G=\mathbb{Z} / 4+\mathbb{Z} / 2$ or $G=Q_{8}$, so assume $n>2$ in the following argument. We have $\left(\left(h_{1}, h_{2}\right) \tau\right)^{-1}(a, \alpha(a))\left(h_{1}, h_{2}\right) \tau=$ $\left(h_{2}^{-1} \alpha(a) h_{2}, h_{1}^{-1} a h_{1}\right)$. Any conjugation of an element in $\mathrm{PGL}_{2}$ fixing $\mathbb{Z} / n$ will send $a$ to either $a$ or $a^{-1}$, so $\alpha^{4}(a)=a$. If $\alpha(a)=a^{-1}$ then, after composing the conjugation of $b$, we can reduce to the case where $\alpha=\mathrm{id}$.

Case 1: $\alpha=\mathrm{id}$. From now on, we change our notation by writing $h_{1} h_{2}^{-1}$ as $t$ and $h_{2}$ as $r$. Now the preceding information reads as follows: $t$ commutes with $D_{2 n}, r$ normalizes $D_{2 n}$, and $r^{2} t \in D_{2 n}$. Now if $t=e$, then we have a composition of group homomorphisms, $f: G \rightarrow \mathrm{PGL}_{2} \times \mathbb{Z} / 2 \rightarrow \mathrm{PGL}_{2}$. If $\operatorname{Ker}(f)=\mathbb{Z} / 2$, then $\tau \in G$ and $G=D_{2 n} \times \mathbb{Z} / 2$. Otherwise, $f$ is an isomorphism from $G$ to its image, which is a polyhedral group containing $D_{2 n}$ as an index-2 subgroup. By 
(*), we have that $\left(\mathbb{F}_{0}, G\right)$ is birational to an action with IFP if and only if $\left(G, G^{0}\right)$ is $\left(D_{4 n}, D_{2 n}\right)$ and $n$ is even.

Suppose $t \neq e$. If $r \in D_{2 n}$, we can write $h$ to be $(-1,1) \tau$. Since $h^{2}=(-1,-1)$ implies that $n$ is even, it follows that $G=G_{4 n}$. When $n$ is divided by $4,(-i, i) \tau$ and $(i, i) \tau$ generate a subgroup as $H$ in $(*)$. On the other hand, $\sum_{G_{16 k+8}}$ is empty, so in this case it is birational with IFP if and only if $n=4 k+2$. If $r \notin D_{2 n}$, then $-r^{2} \in G^{0}$. We can assume that $r$ commutes with $a$ and so $h^{-1}(b, b) h=$ $\left(r^{2} b, r^{2} b\right)$. This implies $r^{2} \in D_{2 n}$, so $n$ is even. Then the same argument as used before shows that $G$ is birational with IFP if and only if $n=4 k+2$.

Case 2: $\alpha$ has order 4. Now we can assume $h_{1}=b t_{1}$ and $h_{2}=t_{2}$, where $t_{i}$ $(i=1,2)$ are commutators of $a$. Hence $\left(\left(h_{1}, h_{2}\right) \tau\right)^{2}=\left(b t_{1} t_{2}, b t_{2}^{-1} t_{1}\right)$, which is never trivial. This is saying that $G$ acts on $F_{0}$ with IFP. Assume $\alpha(a)=a^{q}$; then $q^{2} \equiv-1(\bmod n)$. Let $t_{1} t_{2}=a^{k_{1}}$ and $t_{2}^{2}=a^{k_{2}}$; then $q k_{1}=k_{1}-k_{2}$.

Suppose $n$ is even. This implies that $t_{2}$ is in $D_{2 n}$, and we can choose $t_{2}=e$. That $\operatorname{gcd}(q-1, n)=2$ implies that $k_{1}=0$ or $\frac{n}{2}$. Thus we have

(1) $H_{4 n}$ generated by $a, b$, and $(b, 1) \tau$, and

(2) $I_{4 n}$ generated by $a, b$, and $(b,-1) \tau$,

where $H_{4 n} \cong I_{4 n}$ as abstract groups.

Suppose $n$ is odd. If $t_{2}$ is in $D_{2 n}$, again we assume it is $e$. Since $\operatorname{gcd}(q-1, n)=$ 1 , we know that $t_{1}=e$ and we get $H_{4 n}$. If $t_{2}$ is not in $D_{2 n}$ then we can assume it is -1 , which implies that $t_{1}=-1$. Then we have a group $J_{4 n}$ generated by $a, b$, and $(-b,-1) \tau$, and $J_{4 n}$ is also isomorphic to $H_{4 n}$ as an abstract group.

$$
\text { The Case } S=\mathbb{F}_{e}, e>1
$$

3.10. Proposition. The actions on $\mathbb{F}_{e}(e \geq 1)$ that are birational with IFP are as follows:

(i) when e is even, $G$ are the groups in Proposition 3.3;

(ii) when e is odd, $G \subset \mathbb{C}^{*} \times \mathrm{PGL}_{2}$ are the groups described in (i) with the form $\left(\mathbb{Z} / m k, \mathbb{Z} / m, G_{1}, G_{2}\right)$.

Proof. In fact, $\operatorname{Aut}\left(\mathbb{F}_{n}\right) \cong \operatorname{Aut}(\mathbb{P}(1,1, n)) \cong \mathbb{C}^{n+1}: \mathrm{GL}_{2} / \mu_{n}$; therefore, if a finite group $G \in \operatorname{Aut}\left(\mathbb{F}_{n}\right)$ then $G \in \mathrm{GL}_{2} / \mu_{n}$. Since

$$
1 \rightarrow \mathbb{Z} / 2 \rightarrow \mathbb{C}^{*} \times \mathrm{SL}_{2} \rightarrow \mathrm{GL}_{2} \rightarrow 1,
$$

we have $\mathrm{GL}_{2} / \mu_{n} \cong C^{*} / \mu_{n} \times \mathrm{PGL}_{2} \cong \mathbb{C}^{*} \times \mathrm{PGL}_{2}($ for even $n)$ or $\left(\mathbb{C}^{*} / \mu_{n} \times \mathrm{SL}_{2}\right) /$ $\mathbb{Z} / 2 \cong \mathrm{GL}_{2}$ (for odd $n$ ). Using an argument similar to that used in the previous proof then yields the conclusion. The details are left to the reader.

\section{The Case of $S$ a Nonminimal G-Ruled Surface}

3.11. Proposition. Assume for a minimal action $(S, G)$ that $\pi: S \rightarrow P^{1}$ gives a $G$-equivariant fibration and that $S$ itself is not minimal. Then $(S, G)$ is not birational to any action with IFP. 
Proof. Consider the natural group homomorphism $f: G \rightarrow \operatorname{Aut}(\operatorname{Pic}(S))$. If its kernel $G_{0}$ is not trivial, then by [DI, Prop. 5.5] we know that $S$ is an exceptional conic bundle-in other words, the minimal resolution of the degree- $(2 g+2)$ hypersurface

$$
F_{2 g+2}\left(T_{0}, T_{1}\right)+T_{2} T_{3}=0
$$

in the weighted projective space $\mathbb{P}(1,1, g+1, g+1)$ (cf. the construction of [DI, Sec. 5.2]). The automorphism group of $S$ is an extension of $P$ by $N$, where $P$ is the subgroup of $\mathrm{PGL}_{2}$ leaving the zero sets of $F_{2 g+2}$ invariant and where $N \cong \mathbb{C}^{*}: 2$ is a group of matrices with determinant \pm 1 leaving $T_{2} T_{3}$ invariant (cf. [DI, Prop. 5.3]). The kernel $K$ of $f$ is the intersection of $G$ with $N$ and fixes the coordinates $T_{0}$ and $T_{1}$ as well as the left $T_{2} T_{3}$ invariant. Hence it fixes the curve that is the birational transform of $C: F_{2 g+2}\left(T_{0}, T_{1}\right)+T^{2}=0$ pointwise. Because this curve is of genus $\geq 1$, the action is not birational with IFP.

If $G_{0}=\{e\}$, then by the following proposition we know that $G$ contains an order-2 element fixing a curve of genus $g \geq 1$ pointwise.

3.12. Proposition [DI, Thm. 5.7]. Let $G$ be a minimal finite group of automorphisms of a conic bundle $\varphi: S \rightarrow \mathbb{P}^{1}$ with a set $\Sigma$ of singular fibers. Assume that $G_{0}=e$. Then $k=|\Sigma|>2$, and one of the following cases occurs.

(i) $G=2 \bullet P$, where the central involution $h$ fixes pointwise an irreducible smooth bisection $C$ of $\varphi$ and switches the components in all fibers. The curve $C$ is a curve of genus $g=(k 2) / 2$. The conic bundle projection defines a $g_{2}^{1}$ on $C$ with ramification points equal to singular points of fibers. The group $P$ is isomorphic to the group of automorphisms of $C$ modulo the involution defined by the $g_{2}^{1}$.

(ii) $G \cong 2^{2} \bullet P$, where each nontrivial element $g_{i}$ of the subgroup $2^{2}$ fixes pointwise an irreducible smooth bisection $C_{i}$. The set $\Sigma$ is partitioned into three subsets $\Sigma_{1}, \Sigma_{2}, \Sigma_{3}$ such that the projection $\varphi: C_{i} \mathbb{P}^{1}$ ramifies over $\Sigma_{j}+\Sigma_{k}$, $i \neq j \neq k$. The group $P$ is a subgroup of Aut $\left(\mathbb{P}^{1}\right)$ leaving the set $\Sigma$ (and its partition into three subsets $\Sigma_{i}$ ) invariant.

It remains to study the cases when $(S, G)$ is minimal and $S$ is a smooth del Pezzo surface. For the del Pezzo surfaces of degree 7 and 8, no minimal action exists.

\section{The Case of $S$ the del Pezzo Surface of Degree 6}

3.13. Proposition [DI, Thm. 6.3]. Let $G$ be a minimal subgroup of a del Pezzo surface $S$ of degree 6 . Then $G=H \bullet\langle s\rangle$, where $H$ is a transitive imprimitive finite subgroup of $\mathrm{PGL}_{3}$ and $s$ is the lift of the standard quadratic transformation.

3.14. Proposition. If $(S, G)$ is birational to an action with IFP, then $G$ is $S_{3}$ or $\mathbb{Z} / 2 \times(\mathbb{Z} / n: \mathbb{Z} / 3)$ for $n \geq 1$.

Proof. That $G=H \bullet\langle s\rangle$ acts on $S$ birationally with IFP implies that the same holds for $H$ on $S$, which has a minimal model of $H$ on $\mathbb{P}^{2}$ as an imprimitive action. So $H$ can be only the groups as in Proposition 3.6. 
We claim that the action of $G=\mathbb{Z} / 2 \times S_{3}\left(H=S_{3}\right)$ is not birational to any action with IFP. In fact, the abelian subgroup generated by the lifting of the order-2 element $\rho:\left(x_{0}, x_{1}, x_{2}\right) \rightarrow\left(x_{1}, x_{0}, x_{2}\right)$ and the Cremona transformation $\tau$ is isomorphic to $(\mathbb{Z} / 2)^{2}$. We have $S^{\rho}=\left(x_{0}=x_{1}\right)$ and $S^{\rho \tau}=\left(x_{0} x_{1}=x_{2}^{2}\right)$. This gives a cycle in $\sum_{S}$ satisfying the assumption of Corollary 2.2.

The only remaining case is when $H=\mathbb{Z} / n: \mathbb{Z} / 3$. We claim that the action $(S, G)$ itself is already with IFP. First, we know that any nontrivial element in the subgroup $G_{n, s}$ acts on $S$ with IFP. For an element $g \in G_{n, s}: \mathbb{Z} / 6$ with $g^{6} \in G_{n, s}$, if $g^{6}$ is nontrivial then $g$ acts on $S$ with IFP. Because $n \mid s^{2}-s+1 \equiv 0$ implies $n$ is odd, we know that if $S^{g}$ is not isolated then $g^{3}$ is trivial. Thus, we only need to verify the statement for the case of $G=G_{3,2}: \mathbb{Z} / 6=(\mathbb{Z} / 3)^{2} \times \mathbb{Z} / 2$. However, both the Cremona transformation and any element in $(\mathbb{Z} / 3)^{2}$ fix finite points, so we conclude that the $G_{n, s}: \mathbb{Z} / 6$ action is an action with IFP.

\section{The Case of $S$ the del Pezzo Surface of Degree 5}

For Aut $(S)=S_{5}$, we assume that $S$ is obtained by blowing up four points $(1,0,0)$, $(0,1,0),(0,0,1)$, and $(1,1,1)$.

3.15. Proposition [DI, Thm. 6.4]. Let $(S, G)$ be a minimal del Pezzo surface of degree $d=5$. Then $G=S_{5}, A_{5}, \mathbb{Z} / 5: \mathbb{Z} / 4, \mathbb{Z} / 5: \mathbb{Z} / 2$, or $\mathbb{Z} / 5$.

3.16. Proposition. Let $(S, G)$ be a minimal action on the smooth del Pezzo surface $S$ of degree 5 , and assume that $(S, G)$ is birational to an action with IFP. Then $G=\mathbb{Z} / 5: \mathbb{Z} / 4, \mathbb{Z} / 5: \mathbb{Z} / 2$, or $\mathbb{Z} / 5$.

Proof. For $G=A_{5}$ there is a cycle in $\sum_{S}$ whose edges are birational transforms of lines $\left\{x_{i}+x_{j}=x_{k}\right\}(\{i, j, k\}=\{1,2,3\})$. The vertices are $(1,1,0),(1,0,1)$, and $(0,1,1)$, and each has a noncyclic abelian stabilizer. Then (by Corollary 2.2) we know that $A_{5}$ is not birational to any action with IFP.

Suppose $G=\mathbb{Z} / 5: \mathbb{Z} / 4$. We can represent the elements of $G \cong \mathbb{Z} / 5: \mathbb{Z} / 4=$ $D_{10}: \mathbb{Z} / 2$ as follows:

- $(12345)=\left(x_{0}, x_{1}, x_{2}\right) \rightarrow\left(x_{0}\left(x_{2}-x_{1}\right), x_{2}\left(x_{0}-x_{1}\right), x_{0} x_{2}\right)$;

- $(2354)=\left(x_{0}, x_{1}, x_{2}\right) \rightarrow\left(x_{2}\left(x_{0}-x_{1}\right), x_{2}\left(x_{0}-x_{2}\right), x_{1}\left(x_{0}-x_{2}\right)\right)$.

Then $\sum_{S}$ contains five irreducible curves, any two of which intersect at two identical points $\left(\frac{-1-\sqrt{5}}{2}, \frac{3+\sqrt{5}}{2}, 1\right)$ and $\left(\frac{-1+\sqrt{5}}{2}, \frac{3-\sqrt{5}}{2}, 1\right)$. We can obtain an action on $\mathbb{F}_{0}$ by first blowing up these two points and then contracting the birational transforms of the five curves just listed.

In the last part of this section we will prove that, for any minimal action on a smooth del Pezzo surface of degree $<5$, the group always contains a nontrivial element that fixes a curve of genus $g>0$ pointwise. In particular, this implies the nonexistence of any minimal action on such surfaces that is birational to an action with IFP. First, we study some general theory of a finite group $G$ acting on a smooth surface $S$; then we apply it to the case when $S$ is rational. 
For any nontrivial automorphism $g$ of a surface $S$, by the Lefschetz fixed point formula we have

$$
2-\operatorname{Tr}_{1}(g)+\operatorname{Tr}_{2}(g)=e\left(S^{g}\right)=s+\sum_{j=1}^{t}\left(2-2 g\left(C_{j}\right)\right) .
$$

Here $\operatorname{Tr}_{i}$ denotes the trace of the $g$ acting on the $i$ th singular cohomology, $C_{j}$ the disjoint smooth curves fixed by $g$, and $s$ the number of isolated fixed points. When $S$ is rational, $H^{1}(S)=0$ and $\operatorname{Pic}(S)=H^{2}(S, \mathbb{Z})$. Then, for a group $G$ acting on $S$, we have

$$
\begin{aligned}
\operatorname{rank}\left(\operatorname{Pic}(S)^{G}\right) & =\frac{1}{|G|} \sum_{g \in G} \operatorname{Tr}_{2}(g) \\
& =\frac{1}{|G|}\left(\operatorname{rank}(S)+\sum_{g \in G-\{e\}}\left(s-2+\sum_{j=1}^{t}\left(2-2 g\left(C_{j}\right)\right)\right)\right) .
\end{aligned}
$$

If $(S, G)$ is minimal but $S$ is not a $G$-equivariant conic bundle, then we have $\operatorname{rank}\left(\operatorname{Pic}(S)^{G}\right)=1$.

3.17. Remark. Although the general theory just described is illuminating, in the following proofs we must use the classification results from [DI]. For this reason, we will use their terminology without specific reference. However, it would be nice to find a straightforward argument that does not depend so heavily on the classification results.

\section{The Case of $S$ a del Pezzo Surface of Degree 4}

The reader can check [DI, Sec. 6.4] for all minimal actions $G$ on $S$ a del Pezzo surface of degree 4. Let us summarize the facts we need here. The surface $S$ is isomorphic to a nonsingular surface of degree 4 in $\mathbb{P}^{4}$ given by the equations

$$
F_{1}=\sum_{i=0}^{4} T_{i}^{2}=0 \quad \text { and } \quad F_{2}=\sum_{i=0}^{4} a_{i} T_{i}^{2}=0
$$

where all the $a_{i}$ are distinct. The Weyl group is $W\left(D_{5}\right)=\mathbb{Z} / 2^{4}: S_{5}$, and the automorphism group of $S$ (which is a subgroup of $W\left(D_{5}\right)$ ) always has $(\mathbb{Z} / 2)^{4}$ as a normal subgroup; it is given by changing an even number of signs of the coordinates. So for $A$ an even cardinality subset of $\{0,1,2,3,4\}$ we can compute the fixed locus of $i_{A}$, which is the automorphism of changing the signs of coordinates corresponding to $A$. When $|A|=4$, the fixed locus is an elliptic curve; when $|A|=2$, the fixed locus consists of isolated points. Observe that $(\mathbb{Z} / 2)^{4} \cap G$ can only be $e, i_{a b}$, or $\left\langle i_{a b}, i_{a c}\right\rangle$.

The subgroup $G^{\prime}$ of Aut $(S)$ can be realized as the stabilizer of a set of five skew lines on $S$. Thus $G^{\prime}$ is isomorphic to a group of projective transformations of $\mathbb{P}^{2}$ leaving invariant a set of five points. Because there is a unique conic through these points, the group is isomorphic to a finite group of $\mathrm{PGL}_{2}$ leaving invariant a set of 
five distinct points. It follows that a subgroup leaves invariant a set of five distinct points if and only if it is one of the following groups: $\mathbb{Z} / 2, \mathbb{Z} / 3, \mathbb{Z} / 4, \mathbb{Z} / 5, S_{3}, D_{10}$.

3.18. Proposition [DI, Thm. 6.9]. Let $(S, G)$ be a minimal del Pezzo surface of degree 4. Then $G$ is isomorphic to one of the following groups.

(i) $\operatorname{Aut}(S) \cong(\mathbb{Z} / 2)^{4}$,

$$
(\mathbb{Z} / 2)^{4},(\mathbb{Z} / 2)^{3},(\mathbb{Z} / 2)^{2} .
$$

(ii) $\operatorname{Aut}(S) \cong(\mathbb{Z} / 2)^{4}: \mathbb{Z} / 2$,

$$
\mathbb{Z} / 2 \times \mathbb{Z} / 4, D_{8}, L_{16},(\mathbb{Z} / 2)^{4}: \mathbb{Z} / 2,
$$

and those from case (i).

(iii) $\operatorname{Aut}(S) \cong(\mathbb{Z} / 2)^{4}: \mathbb{Z} / 4$,

$$
8,(\mathbb{Z} / 2)^{2}: \mathbb{Z} / 8,(\mathbb{Z} / 2)^{4}: \mathbb{Z} / 4,
$$

and those from cases (i) and (ii).

(iv) $\operatorname{Aut}(S) \cong(\mathbb{Z} / 2)^{4}: S_{3}$,

$$
(\mathbb{Z} / 2)^{2} \times \mathbb{Z} / 3, \mathbb{Z} / 2 \times A_{4},(\mathbb{Z} / 2)^{4}: \mathbb{Z} / 3,(\mathbb{Z} / 2)^{4}: S_{3},
$$

and those from cases (i) and (ii); here $A_{4} \cong(\mathbb{Z} / 2)^{3}: \mathbb{Z} / 3$.

(v) $\operatorname{Aut}(S) \cong(\mathbb{Z} / 2)^{4}: D_{10}$,

$$
(\mathbb{Z} / 2)^{4}: D_{10},(\mathbb{Z} / 2)^{4}: \mathbb{Z} / 5,
$$

and those from cases (i) and (ii).

We claim that each of these minimal actions contains an element of the form $i_{a b c d}$. Otherwise, $K:=(\mathbb{Z} / 2)^{4} \cap G$ can only be $e, i_{a b}$, or $\left\langle i_{a b}, i_{a c}\right\rangle$. Since none of these actions are minimal, we conclude that $G \subsetneq(\mathbb{Z} / 2)^{4}$ and hence the image $G^{\prime}$ of $G$ in $S_{5}$ is nontrivial. Also, we need only look at the cases where $|K| \leq 4$. In case (ii), $G=\mathbb{Z} / 2 \times \mathbb{Z} / 4$ implies $K=\left\langle i_{01}, i_{0123}\right\rangle$ while $G=D_{8}$ implies $K=$ $\left\langle i_{0123}, i_{0124}\right\rangle$. In case (iii), $G=\mathbb{Z} / 2 \times \mathbb{Z} / 4$ implies $K=\left\langle i_{0123}\right\rangle$; in case (iv), $G=$ $(\mathbb{Z} / 2)^{2} \times \mathbb{Z} / 3$ implies $K=\left\langle i_{0123}, i_{0124}\right\rangle$.

Thus we conclude as follows.

3.19. Proposition. Any minimal action on a del Pezzo surface $S$ of degree 4 is not birational to an action with IFP.

\section{The Case of S a Cubic Surface}

All the possible minimal actions on a smooth cubic surface are classified in [DI, Sec. 6.5]. There exist cyclic groups whose generator fixes an elliptic curve pointwise:

- $\left[t_{0}, t_{1}, t_{2},-t_{3}\right]$,

$$
F=T_{3}^{2} L_{1}\left(T_{0}, T_{1}, T_{2}\right)+T_{0}^{3}+T_{1}^{3}+T_{2}^{3}+\alpha T_{0} T_{1} T_{2} ;
$$

the generator is of type $4 A_{1}$. 
- $\left[t_{0}, t_{1}, t_{2}, \varepsilon_{3} t_{3}\right]$,

$$
F=T_{0}^{3}+T_{1}^{3}+T_{2}^{3}+T_{3}^{3}+\alpha T_{0} T_{1} T_{2} ;
$$

the generator is of type $3 A_{1}$.

We will check that, for all minimal actions $(S, G), G$ always contains elements of one of the listed types.

Table 4 of [DI] gives all types of cubic surfaces and their automorphic groups. Because there are some specializations (IV $\rightarrow$ III, IV $\rightarrow$ I, VI, VIII, IX $\rightarrow$ I, $\mathrm{XI} \rightarrow \mathrm{X}$ ), it suffices to consider surfaces of types I, II, III, V, VII, and X.

3.20. Proposition [DI, Thm. 6.14]. Let $G$ be a minimal subgroup of automorphisms of a nonsingular cubic surface of type I, II, III, V, VII, or X. Then G is isomorphic to one of the following groups, where the $n$ in $G(n)$ denotes $n$ different conjugacy classes.

(i) $G$ is a subgroup of automorphisms of a surface of type I:

$$
\begin{gathered}
S_{4}(3), S_{3}(2), S_{3} \times \mathbb{Z} / 2, S_{3} \times \mathbb{Z} / 3(2),(\mathbb{Z} / 3)^{2}:(\mathbb{Z} / 2)(2),(\mathbb{Z} / 3)^{2}:(\mathbb{Z} / 2)^{2}, \\
H_{3}(3): \mathbb{Z} / 2, H_{3}(3),(\mathbb{Z} / 3)^{3}:(\mathbb{Z} / 2)(2),(\mathbb{Z} / 3)^{3}:(\mathbb{Z} / 2)^{2}(2), \\
(\mathbb{Z} / 3)^{3}: \mathbb{Z} / 3,(\mathbb{Z} / 3)^{3}: S_{3},(\mathbb{Z} / 3)^{3}: D_{8},(\mathbb{Z} / 3)^{3}: S_{4},(\mathbb{Z} / 3)^{3}: \mathbb{Z} / 4, \\
(\mathbb{Z} / 3)^{3},(\mathbb{Z} / 3)^{2}(3),(\mathbb{Z} / 3)^{2} \times \mathbb{Z} / 2, \mathbb{Z} / 9, \mathbb{Z} / 6(2), \mathbb{Z} / 3 .
\end{gathered}
$$

(ii) $G$ is a subgroup of automorphisms of a surface of type II:

$$
S_{5}, S_{4} \text {. }
$$

(iii) $G$ is a subgroup of automorphisms of a surface of type III:

$$
H_{3}(3): \mathbb{Z} / 4, H_{3}(3): \mathbb{Z} / 2, H_{3}(3), S_{3} \times \mathbb{Z} / 3, S_{3},(\mathbb{Z} / 3)^{2}, \mathbb{Z} / 12, \mathbb{Z} / 6, \mathbb{Z} / 3 .
$$

(iv) $G$ is a subgroup of automorphisms of a surface of type $I V$ :

$$
H_{3}(3): 2, H_{3}(3), S_{3}(2), 3 \times S_{3}(2),(\mathbb{Z} / 3)^{2}(2), \mathbb{Z} / 6, \mathbb{Z} / 3 .
$$

(v) $G$ is a subgroup of automorphisms of a surface of type $V$ :

$$
S_{4}, S_{3} .
$$

We will discuss these case by case but in a slightly different order.

(1) The cubic surface of type I:

$$
T_{0}^{3}+T_{1}^{3}+T_{2}^{3}+T_{3}^{3}=0
$$

Its automorphism group is $(\mathbb{Z} / 3)^{3}: S_{4}$. The factor of $(\mathbb{Z} / 3)^{3}$ comes from sending $\left(T_{0}, T_{1}, T_{2}, T_{3}\right)$ to $\left(\varepsilon_{3}^{a} T_{0}, \varepsilon_{3}^{b} T_{1}, \varepsilon_{3}^{c} T_{2}, \varepsilon_{3}^{b} T_{3}\right)$, which we denote as $[a, b, c, d]$. We require

$$
a+b+c+d=0 \text { in } \mathbb{F}_{3} .
$$

Then, up to symmetry, there are three different types of 1-dimensional space in $\mathbb{F}_{3}^{3}$, which are represented by 


$$
[1,2,0,0],[1,1,1,0],[1,1,2,2] \text {. }
$$

The different classes of 2-dimensional spaces of $\mathbb{F}_{3}^{3}$ are given by the orthogonal complement in $\left(\mathbb{F}^{3}\right)^{3}$ with respect to the dot-product pairing on $\mathbb{F}_{3}^{4}$. Let $K=$ $G \cap(\mathbb{Z} / 3)^{3}$ and $\operatorname{dim}_{\mathbb{F}_{3}} K=k$.

(a) For $k=0, G$ is either $S_{4}$ or $S_{3}$. When $G=S_{4}$, it has three different conjugacy classes, each of which has the six elements of type $4 A_{1}$. When $G=S_{3}$, it has three elements of type $4 A_{1}$.

(b) For $k=1$, if $K=\langle[1,1,2,2]\rangle$ then the action is not minimal; if $K=$ $\langle[1,1,1,0]\rangle$ then the generator of $K$ is of type $3 A_{2}$; and if $K=\langle[0,0,1,2]\rangle$ then $G=S_{3}$ and contains three elements of type $4 A_{1}$.

(c) For $k=2$, if $K$ is an orthogonal complement of $[1,1,2,2]$ then the action is not minimal. But for the other two cases discussed in [DI], $K$ contains element of type $[1,1,1,0]$, which is indeed of type $3 A_{1}$.

(d) For $k=3, K$ contains an element of type $3 A_{2}$.

(2) The cubic surface of type II:

$$
T_{0}^{2} T_{1}+T_{1}^{2} T_{2}+T_{2}^{2} T_{3}+T_{3}^{2} T_{0}=0 .
$$

The surface is isomorphic to the Clebsch diagonal cubic surface in $\mathbb{P}^{4}$ given by the equations

$$
\sum_{i=0}^{4} T_{i}^{3}=\sum_{i=0}^{4} T_{i}=0 .
$$

The group $S_{5}$ acts by permuting the coordinates. The transposition (12) is of type $4 A_{1}$, and the minimal action (which has $G=S_{5}$ or $S_{4}$ ) always contains transpositions.

(3) For the cubic surface of type IV:

$$
T_{0}^{3}+T_{1}^{3}+T_{2}^{3}+T_{3}^{3}+6 a T_{1} T_{2} T_{3}=0,
$$

where the parameter $a$ satisfies $a a^{4} \neq 0,8 a^{3} \neq 1$, and $20 a^{3}+8 a^{6} \neq 1$. The surface's automorphic group is $H_{3}(3): 2$, and when $a=1$ its specialization is of type I. Given a smooth family of cubic $G$-surfaces $\left(S_{t}, G\right)$, both the minimality of the action and the type of an element $g \in G$ are invariant. Hence, from the argument for type I, all the minimal actions on a type IV cubic surface are not birational to actions with IFP.

(4) For the cubic surface of type III:

$$
T_{0}^{3}+T_{1}^{3}+T_{2}^{3}+T_{3}^{3}+6 a T_{1} T_{2} T_{3}=0,
$$

where $20 a^{3}+8 a^{6}=1$. It is obviously a specialization of type IV and there are two new groups, $H_{3}(3): 4$ and $\mathbb{Z} / 12$; both of them contain elements of type $3 A_{1}$.

(5) The cubic surface of type V:

$$
T_{0}^{3}+T_{0}\left(T_{1}^{2}+T_{2}^{2}+T_{3}^{2}\right)+a T_{1} T_{2} T_{3}=0,
$$

where $9 a^{3} \neq 8 a$ and $8 a^{3} \neq 1$. Its automorphic group is $S_{4} \cong(\mathbb{Z} / 2)^{2}: S_{3}$, which acts by permuting the coordinates $T_{1}, T_{2}, T_{3}$ and then multiplying them by -1 , 
leaving the monomial $T_{1} T_{2} T_{3}$ unchanged. The only minimal action is when $G=$ $S_{4}$ or its subgroup $S_{3}$. We remark that in the representation just displayed, any transposition contained in $S_{3}$ is of type $4 A_{1}$.

If a cubic surface of any other type has some minimal actions, then it can be specialized to one of the preceding types. As in (3), we conclude that none of them is birational to an action with fixed points. Hence we have the following statement.

3.21. Proposition. No minimal action on a cubic surface $S$ is birational to an action with IFP.

\section{The Case of S a del Pezzo Surface of Degree 2}

All the minimal actions on a del Pezzo surface $S$ of degree 2 are discussed in [DI, Sec. 6.6]. Since $S$ can be written as a double curve of $\mathbb{P}^{2}$ branched over a quartic curve $B$, we know that there is a homomorphism $\operatorname{Aut}(S) \rightarrow \operatorname{Aut}(B)$ with the kernel generated by the Geiser involution. There also exist cyclic groups whose generator fixes an elliptic curve pointwise:

- $\left[t_{0}, t_{1},-t_{2}, t_{3}\right]$,

$$
F=T_{3}^{2}+T_{2}^{4}+T_{2}^{2} L_{2}\left(T_{0}, T_{1}\right)+L_{4}\left(T_{0}, T_{1}\right) ;
$$

the generator is of type $4 A_{1}$.

- $\left[t_{0}, t_{1}, \varepsilon_{3} t_{2}, t_{3}\right]$,

$$
F=T_{3}^{2}+T_{2}^{3} L_{1}\left(T_{0}, T_{1}\right)+L_{4}\left(T_{0}, T_{1}\right) ;
$$

the generator is of type $3 A_{1}$.

It therefore suffices to check that, for any minimal action $(S, G), G$ contains either the Geiser involution or an element of type $4 A_{1}$ or $3 A_{1}$. By [DI, Lemma 6.16], if $g$ is an element of order 4 then $g^{2}$ is either an element of type $4 A_{1}$ or the Geiser involution, depending on whether the image of $g$ in $\operatorname{Aut}(B)$ is (respectively) of order 4 or 2; if $g$ is an element of order 6 , then either $g^{2}$ is of type $3 A_{1}$ or $g^{3}$ is the Geiser involution, depending on whether the image of $g$ in $\operatorname{Aut}(B)$ is (respectively) of order 6 or 3 . Thus, a minimal group containing an element of order 4 or 6 is not birational with IFP.

3.22. Proposition [DI, Thm. 6.17]. Let $G$ be a minimal group of automorphisms of a del Pezzo surface of degree 2. Then $G$ either contains the Geiser involution or is equal to one of the following minimal lifts of a subgroup of $\operatorname{Aut}(B)$.

(i) Type I: $L_{2}(7), S_{4}(2), D_{8}$.

(ii) Type II: $\mathbb{Z} / 4^{2}: S_{3}(2), S_{4}(2),(\mathbb{Z} / 4)^{2}: \mathbb{Z} / 3, A_{4},(\mathbb{Z} / 4)^{2}: \mathbb{Z} / 2(3)$,

$$
M_{16}, A S_{16}(2), D_{8},\left(\mathbb{Z} / 4_{2}\right),(\mathbb{Z} / 2 \times \mathbb{Z} / 4)(2), \mathbb{Z} / 4,
$$

where $A S_{16}$ is the group of a presentation,

$$
a^{4}=b^{2}=c^{2}=[a, b]=1, \quad[c, b] a^{-2}=[c, a]=1,
$$

and $M_{16}$ is the group of a presentation,

$$
a^{8}=b^{2}=1, \quad[a, b] a^{4}=1 .
$$


(iii) Type III:

$$
\mathbb{Z} / 4 \bullet A_{4}(2), D_{8}: 3, A S_{16}(2), D_{8}, \mathbb{Z} / 12, \mathbb{Z} / 6, \mathbb{Z} / 2 \times \mathbb{Z} / 4, \mathbb{Z} / 4 .
$$

(iv) Type $I V: S_{4}, D_{8}$.

(v) Type $V$ : $A S 16(2), D_{8}, \mathbb{Z} / 2 \times \mathbb{Z} / 4(2), \mathbb{Z} / 4$.

(vi) Type VII: $D_{8}$.

(vii) Type VIII: $\mathbb{Z} / 6$.

We have the following specializations:

$$
\mathrm{IX} \rightarrow \mathrm{IV} \rightarrow \mathrm{I}, \mathrm{II}, \mathrm{XII} \rightarrow \mathrm{X} \rightarrow \mathrm{VII} \rightarrow \mathrm{V} \rightarrow \mathrm{II}, \mathrm{III}, \mathrm{XI} \rightarrow \mathrm{VIII} \rightarrow \text { III }
$$

Hence we need only discuss the minimal groups that do not contain the Geiser involution and only for surfaces of type I, II, or III.

(1) For the degree-2 del Pezzo surface of type I:

$$
T_{3}^{2}+T_{0}^{3} T_{1}+T_{1}^{3} T_{2}+T_{2}^{3} T_{0}=0 .
$$

The order-2 element in $L_{2}(7)$ is of type $4 A_{1}$, and $S_{4}$ and $D_{8}$ contain elements of order 4.

(2) For the degree-2 del Pezzo surface of type II:

$$
T_{3}^{2}+T_{0}^{4}+T_{1}^{4}+T_{2}^{4}=0 .
$$

All the groups contain an element of order 4 .

(3) For the degree-2 del Pezzo surface of type III:

$$
T_{3}^{2}+T_{2}^{4}+T_{0}^{4}+a T_{0}^{2} T_{1}^{2}+T_{1}^{4}=0 \quad\left(a^{2}=-12\right) .
$$

The groups contain an element whose order is either 4 or 6 .

Thus we conclude as follows.

3.23. Proposition. No minimal action on a del Pezzo surface $S$ of degree 2 is birational to an action with IFP.

\section{The Case of $S$ a del Pezzo Surface of Degree 1}

For the remaining cases of del Pezzo surfaces of degree 1, the approach is similar to the case of cubic surfaces. We will only sketch the proof and leave the details to the reader.

All the minimal actions on a del Pezzo surface $S$ of degree 1 are discussed in [DI, Sec. 6.7]. Any $S$ can be written as a degree-6 hypersuface in the weighted projective space $\mathbb{P}(1,1,2,3)$ with the equation

$$
S: T_{3}^{2}+T_{2}^{3}+T_{2} L_{4}\left(T_{0}, T_{1}\right)+L_{6}\left(T_{0}, T_{1}\right)=0 .
$$

The order-2 element of $G$ is one of the following:

(1) the Bertini involution $\left[t_{0}, t_{1}, t_{2},-t_{3}\right]$, which fixes a genus- 4 curve pointwise;

(2) $\left[i t_{0},-i t_{1},-t_{2}, i t_{3}\right]$, where $F_{4}=F_{2}\left(T_{0}^{2}, T_{1}^{2}\right) \neq 0$ and $F_{6}=F_{3}\left(T_{2}^{0}, T_{1}^{2}\right)$, which fixes the genus-1 curve $S \cap\left(t_{0}=0\right)$ pointwise; or 
(3) $\left[-t_{1}, t_{0},-t_{2}, i t_{3}\right]$, where $F_{4}=a\left(T_{0}^{4}+T_{1}^{4}\right)+b T_{0}^{2} T_{1}^{2}$ and $F_{6}=a\left(T_{0}^{6} T_{1}^{6}\right)+$ $b T_{0} T_{1}\left(T_{0}^{4}+T_{1}^{4}\right)$, which fixes the genus-1 curve $S \cap\left(t_{1}=i t_{0}\right)$ pointwise.

Therefore, an action that is birational with IFP cannot contain any order- 2 elements, which means that the order of the group is odd. We can also assume that $G$ does not contain the order-3 element $\left[t_{0}, t_{1}, \varepsilon_{3} t_{2}, t_{3}\right]$ because it fixes a genus- 2 curve pointwise. Then there are only a few cases remaining.

(1) Types I, II, VII, XV: no such group exists.

(2) Types IV, VIII: here $\mathbb{Z} / 5$ is generated by $\left[t_{0}, \varepsilon_{5} t_{1}, t_{2}, t_{3}\right]$, which fixes a genus- 1 curve pointwise.

Thus we conclude with the following result.

3.24. Proposition. No minimal action on a del Pezzo surface $S$ of degree 1 is birational to an action with IFP.

\section{Group Action on log del Pezzo Surfaces}

In this section, we aim to prove Theorem 1.5: given a finite group $G$ and assuming $G$ can act on a rational surface $\tilde{S}$ containing (at worst) quotient singularities giving an action with only IFP, we would like to determine whether $\tilde{S}$ can be chosen to be a log del Pezzo surface. The idea is to run the equivariant minimal model program for the pair $(S, G)$ (see e.g. [KoM] for the general theory of minimal model program). It is well known that a normal surface singularity is $k l t$ if and only if it is a quotient singularity (cf. [KoM, 4.18]). Thus, if we start with a surface that contains (at worst) quotient singularities and run the minimal model program, then after a sequence of divisorial contractions we still have a surface of the same type singularities.

4.1. LemMA. If $(S, G)$ is an action with IFP and if $R \cong S / G$, then running the equivariant minimal model program for $(S, G)$ is equivalent to running the (ordinary) minimal model program for $R$.

Proof. The morphism $\pi: S \rightarrow R$ is finite, $\overline{N E}(S)^{G}=\overline{N E}(R)$, and $\operatorname{Pic}(S)^{G}=$ $\operatorname{Pic}(R)$. Hence it suffices to prove $\pi^{*}\left(n K_{R}\right)=n K_{S}$ for some integer $n$ such that $n K_{R}$ is Cartier. In fact, after removing those isolated branched points, $\pi$ is an étale morphism, so the equality holds in this case. We can therefore conclude that $\pi^{*}\left(n K_{R}\right)=n K_{S}$, since this is an equality of divisors.

4.2. Proposition. Consider the groups $G$ that can act on a smooth rational surface $\tilde{S}$ such that it is birational to an action $(S, G)$ with IFP and $K_{S}$ is not $\mathbb{Q}$-effective. These groups are precisely those that can act on some del Pezzo surfaces with IFP. In other words, they are precisely all quotient groups of $\pi_{1}$ of smooth loci of log del Pezzo surfaces.

Proof. For a surface with only quotient singularities, we know that the $\mathbb{Q}$-effectivity of the canonical class is equivalent to the pseudo-effectivity. Let $R$ be a log 
del Pezzo surface, $G$ a finite quotient group of $\pi_{1}\left(R^{\mathrm{sm}}\right)$, and $S$ the corresponding cover over $R$, which is branched at finite points. Now $(S, G)$ gives us an action with IFP. Because $S$ is also a log del Pezzo surface, $K_{S}$ is not pseudo-effective.

Conversely, we start with $(S, G)$ an action with IFP. Take $R \cong S / G$. We know that $K_{R}$ is not pseudo-effective, either. Running a log minimal model program for $R$ yields

$$
R=R_{0} \rightarrow R_{1} \rightarrow R_{2} \rightarrow \cdots \rightarrow R_{n},
$$

where $R$ and $R_{i}$ are birational. The minimal model program preserves the noneffectivity assumption regarding the $\mathbb{Q}$-divisor $K_{R}$. Thus it terminates with a Fano contraction to a lower-dimensional variety. If it contracts to a point, which is equivalent to saying that $\rho\left(R_{n}\right)=1$, then $R_{n}$ is a log del Pezzo surface and so the group $G$ is a quotient group of $\pi_{1}\left(R^{\mathrm{sm}}\right)$, which itself is a quotient group of $\pi_{1}\left(R_{n}^{\mathrm{sm}}\right)$ by [KMc, 7.3]. Otherwise, $R_{n}$ contracts to $\mathbb{P}^{1}$. By Lemma 4.1, the corresponding $G$-equivariant minimal model program,

$$
S=S_{0} \rightarrow S_{1} \rightarrow S_{2} \rightarrow \cdots \rightarrow S_{n},
$$

gives a contraction from $S_{n}$ to $\mathbb{P}^{1}$ that is a $G$-equivariant fibration. Let $G_{0}$ be the kernel of the natural group homomorphism $\rho:\left.G \rightarrow G\right|_{\mathbb{P}^{1}}$. Any element $g_{0} \in G_{0}$ acts on every fiber; in particular, the set of fixed points is nonempty for every fiber. Hence $g_{0}$ will fix some curves pointwise. Since $\left(S_{n}, G\right)$ is an action with IFP, we conclude that $G_{0}$ is trivial and so $G$ is a subgroup of $\mathrm{PGL}_{2}(\mathbb{C})$. But any such group can act diagonally on $\mathbb{P}^{1} \times \mathbb{P}^{1}$, giving an action with IFP.

The following criterion is useful for proving noneffectivity.

4.3. LemMA. Let $S$ be a projective surface with a $G$ action that is birational to an action with IFP. Suppose there exists a G-birational proper morphism $\phi: \bar{S} \rightarrow S$ with the following property: for any $\mathbb{Q}$-divisor $E$ with supports on $\sum_{\bar{S}}$, we have $\kappa\left(K_{\bar{S}}+E\right)=-\infty$ provided $\lfloor E\rfloor \leq 0$. Then there exsits a birational $G$-model $\left(S^{\prime}, G\right)$ of $(S, G)$ that satisfies

- $\left(S^{\prime}, G\right)$ is an action with IFP and

- $\kappa\left(K_{S^{\prime}}\right)=-\infty$.

Proof. We can assume $\bar{S}=S$. By the argument in $\operatorname{Section} 2$, from $(S, G)$ we can construct an action $\left(S^{\prime}, G\right)$ with IFP and such that, if $\left(S^{*}, G\right)$ is a common resolution of $S$ and $S^{\prime}$,

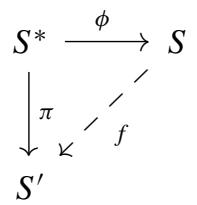

then the exceptional divisors of $\pi$ consist only of curves in $\sum_{S}$ and the exceptional curves of $\phi$. 
Let $\pi^{*}\left(K_{S^{\prime}}\right)=K_{S^{*}}+E+F$, where $\operatorname{Supp}(E)$ is in the birational transform of $\sum_{S}$ and $F$ is exceptional for $\phi$. Then from $\lfloor E+F\rfloor \leq 0$ it follows that $\lfloor E\rfloor \leq 0$. Hence, for any $m \in \mathbb{N}$,

$$
\begin{aligned}
h^{0}\left(m K_{S^{\prime}}\right) & =h^{0}\left(m\left(K_{S^{*}}+E+F\right)\right) \\
& \leq h^{0}\left(m \phi_{*}\left(K_{S^{*}}+E+F\right)\right)=h^{0}\left(m\left(K_{S}+\phi_{*} E\right)=0 .\right.
\end{aligned}
$$

4.4. Lemma. The action $\left(\mathbb{P}^{2}, G\right)$ as in Example 3.5 is not birational to any action on a log del Pezzo surface $(S, G)$ with IFP.

Proof. By way of contradiction, if there is such a surface $S^{\prime}$ then its minimal resolution $\pi: S^{*} \rightarrow S^{\prime}$ has an equivariant morphism $\phi$ to $\mathbb{P}^{2}$, and $\pi$ contracts the birational transform of the lines $\left\{x_{i}=\varepsilon_{3}^{k} x_{j}\right\}$ :

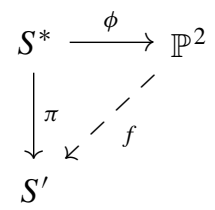

The surface $S^{\prime}$ contains only quotient singularities. Therefore, in the exceptional locus of the morphism $\pi: S^{*} \rightarrow S^{\prime}$, no three irreducible components can intersect at an identical point, which implies that no three components of the birational transform of $\sum_{\mathbb{P}^{2}}$ on $S^{*}$ can intersect at an identical point. Hence the morphism $\phi: S^{*} \rightarrow \mathbb{P}^{2}$ must factor through the surface that we achieve by blowing up the twelve points $\left\{\left(1, \varepsilon_{3}^{i}, \varepsilon_{3}^{j}\right),(1,0,0),(0,1,0),(0,0,1)\right\}$ on $\mathbb{P}^{2}$, where $0 \leq i, j \leq 2$. Then, to get $S^{*}$ from $\mathbb{P}^{2}$, we must blow up at least four points on each of the nine lines $\left\{x_{i}=\varepsilon_{3}^{k} x_{j}\right\}$. Thus the self-intersection numbers of the birational transform of these lines on $S^{*}$ are less than or equal to -3 . If we write $K_{S^{*}}+\sum_{i} a_{i} E_{i}=$ $K_{S}$ with $0<a_{i}<1$, then the coefficients of these lines are greater than or equal to $\frac{1}{3}$ (cf. [A, 2.17]). Hence, for $m$ divisible enough we have

$$
H^{0}\left(S, m K_{S}\right)=H^{0}\left(m\left(K_{S^{*}}+\sum_{i} a_{i} E_{i}\right)\right)=H^{0}\left(\mathbb{P}^{2}, m \phi_{*}\left(K_{S^{*}}+\sum_{i} a_{i} E_{i}\right)\right) .
$$

The second equality holds because $K_{S^{*}}+\sum_{i} a_{i} E_{i}-\phi^{*} \phi_{*}\left(K_{S^{*}}+\sum_{i} a_{i} E_{i}\right) \geq$ 0 , which is implied by the nefness of $-\left(K_{S^{*}}+\sum_{i} a_{i} E_{i}\right)$ (see [KoM, 3.39]). But thanks to our computation of $a_{i}$, we know that $\phi_{*}\left(K_{S^{*}}+\sum_{i} a_{i} E_{i}\right) \geq K_{\mathbb{P}^{2}}+$ $\frac{1}{3} \sum_{i=1}^{9} L_{i} \geq 0$, where the $L_{i}$ are divisors of the nine lines $\left\{x_{i}=\varepsilon_{3}^{k} x_{j}\right\}$.

Proof of Theorem 1.5. By Proposition 4.2 it suffices to prove that, given an action $(S, G)$ as in (i)-(iv) of Theorem 1.2 , we can choose $(\bar{S}, G)$ in the same $G$-birational class satisfying the conditions of Lemma 4.3. According to the previous example, we know that groups containing $(\mathbb{Z} / 3)^{2}: \mathbb{Z} / 2$ cannot act on any log del Pezzo surfaces with IFP. We check the remaining cases from Section 3 as follows.

For Proposition 3.3, after blowing up the origin we obtain a ruled surface $\mathbb{F}_{1}$, and then $\sum_{\mathbb{F}^{1}}$ consists of two sections and a set of fibers. Denote the fiber class 
as $L$. Then $\left(K_{\mathbb{F}_{1}}+E\right) \cdot L<0$, since the coefficients of the sections in $E$ are all less than 1 . Since $|L|$ is a covering family, $K_{\mathbb{F}_{1}}+E$ is not pseudo-effective.

For Proposition $3.6, \mathbb{Z} / n: \mathbb{Z} / 3$ acts on $\mathbb{P}^{2}$ with IFP. For $S_{3}$, blow up the intersection point $(1,1,1)$ of curves in $\sum_{\mathbb{P}^{2}}$; then we can argue as in the previous case.

For Proposition 3.8, if $G$ is $G_{1} \times G_{2}$ then one of them, say $G_{1}$, is cyclic $\left(\left|G_{1}\right|,\left|G_{2}\right|\right.$ are coprime). So $\sum_{\mathbb{F}^{0}, G}$ contains at most two sections for the corresponding fibration. Denoting the class of the fiber as $L$, we have $\left(K_{\mathbb{F}_{0}}+E\right) \cdot L<0$, which implies that $K_{\mathbb{F}_{0}}+E$ is not pseudo-effective. For general $G=\left(G_{1}, H_{1}, G_{2}, H_{2}\right)_{\alpha}$, we can argue in the same way because $\sum_{\mathbb{F}^{0}, G}=\sum_{\mathbb{F}^{0}, H_{1} \times H_{2}}$. If $G: G^{0}=2$ then the only new groups are $F_{4 n}, G_{4 n}$, and $H_{4 n}$. For these cases, the $\sum_{G}$ are always empty.

For Proposition 3.14 , by the proof there we know that, for $\mathbb{Z} / 2 \times(\mathbb{Z} / n: \mathbb{Z} / 3)$, the surface $S$ itself gives a model of action with IFP. For $S_{3}$, it is equivariantly birational to an action on $\mathbb{P}^{2}$ (cf. [DI, 8.1]).

For Proposition 3.16, as discussed before, $(S, G)$ is birational to an action on $\mathbb{F}_{0}$.

\section{5. $\pi_{1}$ of Smooth Loci of log del Pezzo Surfaces}

In the previous section, we give a list containing precisely $\pi_{1}$ of smooth points of log del Pezzo surfaces and all their quotient groups. In this section, we aim to determine which of these groups can be actual fundamental groups. In other words, for a given $G$, we want to construct a $\log$ del Pezzo surface $S$ such that $G$ acts on it with IFP and $\pi_{1}\left(S^{\mathrm{sm}}\right)=e$.

5.1. Proposition. Every group $G$ in (iii) and (iv) of Theorem 1.2 is $\pi_{1}$ of smooth points of some log del Pezzo surface.

Proof. We observe that every group $G$ in (iii) and (iv) of Theorem 1.2 has the following property: for the action $(S, G)$ arising from the classification in Section 3 , $\sum_{S, G}$ contains at most one irreducible curve and $\pi_{1}\left(S \backslash \sum_{S, G}\right)=\{e\}$. Blow up a general orbit of $G$ on $\sum_{S, G}$ and then contract its birational transform; this yields an equivariant model $(\tilde{S}, G)$ such that:

(1) $G$ acts on $\tilde{S}$ with IFP;

(2) $\tilde{S}$ is a log del Pezzo surface; and

(3) $\tilde{S}^{\text {sm }}$ contains $S \backslash \sum_{S, G}$ as an open set.

We thus conclude that $\pi_{1}\left(\tilde{S}^{\mathrm{sm}}\right)=\{e\}$ and that $G$ can be $\pi_{1}$ of smooth points of some log del Pezzo surface.

The remaining cases are subgroups of $\mathrm{GL}_{2}(\mathbb{C})$ or $\mathrm{PGL}_{2}(\mathbb{C}) \times \mathrm{PGL}_{2}(\mathbb{C})$.

5.2. Proposition. Given a group $G$ as in Proposition 3.3 (resp. Proposition 3.8), if $\tilde{G}$ (resp. $\left.G=G^{\prime}\right)$ has the form $\left(\mu_{m k}, \mu_{m}, G_{1}, G_{2}\right)$, then it is a fundamental group of some log del Pezzo surface. 
Proof. If $m=1$, then $G$ is either polyhedral or binary polyhedral. For any binary polyhedral group $G$, consider its action on $\mathbb{P}^{2}$ that factors through $\mathrm{SL}_{2}(\mathbb{C})$. The only possible component of $\sum_{\mathbb{P}^{2}, G}$ is the infinite line $L$. Blowing up a $G$-orbit on $L$ and then contracting $L$, we get a pair $(S, G)$ with IFP. Because $S^{\text {sm }}$ contains $\mathbb{C}^{2}$ as an open set, $\pi_{1}\left(S^{\mathrm{sm}}\right)=\{e\}$.

From now on we assume $m \neq 1$. In the case of Proposition 3.3, we blow up the original point and assume that we always have $S=\mathbb{F}_{e}$. Then the configuration of $\sum_{S, F}$ is as follows.

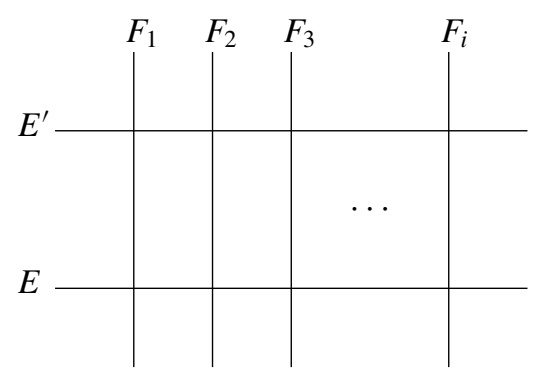

The vertical lines in the diagram are fibers of $\mathbb{F}_{e}$. We will perform the following sequence of $G$-birational operations on $\mathbb{F}_{e}$, which terminates with a $G$-surface $S$ satisfying $\pi_{1}\left(S^{\mathrm{sm}}\right)=e$.

Step 1. We construct a birational model $(S, G)$ such that $\sum_{S, G}$ does not contain any vertical lines.

The way to construct $S$ is as follows. Assuming that $E^{2} \leq 0$, we first equivariantly blow up the intersection points of the $F_{i}$ and $E$ and then contract the $F_{i}$. By Lemma 2.3, we know that, after a finite number of such operations at each intersection point, we will have a $G$-surface $S=\mathbb{F}_{r}$ such that $\sum_{S, G}$ does not contain any fiber.

Step 2. We construct a model $S$ as in Step 1 with the additional property that $-q \leq E^{2}<0$, where $q$ is the length of the $G$-orbit of a general point on $E^{\prime}$.

The way to construct $S$ is similar to Step 1. Assume that $E^{2}=-r<0$ and $\left(E^{\prime}\right)^{2}=r$. We choose a general point $x$ on $E^{\prime}$ so that its stabilizer $G_{x}$ is precisely the subgroup whose elements fix $E^{\prime}$ pointwise; then $q=|G| /\left|G_{x}\right|$. Now blow up these $q$ points and contract the birational transforms of the fibers that pass through them. We have a new ruled surface with $E^{2}=-r+q$ and $\left(E^{\prime}\right)^{2}=r-q$. By the generality of the $q$ points on $E^{\prime}$, we know that $G$ acts on this new surface with $\sum=\left\{E, E^{\prime}\right\}$.

Step 3. There are two cases to deal with.

Case $A:-q<E^{2}$. In this case we first blow up a generic orbit on $E^{\prime}$ and then contract $E$ and $E^{\prime}$ (the contractabilty of $E^{\prime}$ follows from the assumption $-q<$ $\left.E^{2}\right)$. The resulting surface $S$ is a $\log$ del Pezzo surface on which $G$ acts with IFP (see the last part of Step 2) and $\operatorname{Pic}(S)^{G}=\mathbb{Z}$. Then, applying the computation in $[\mathrm{Mu}]$, we can easily conclude that $\pi_{1}\left(S^{\mathrm{sm}}\right)=\{e\}$. 
Case B: $-q=E^{2}$. Again we start by choosing a general orbit of $q$ points on $E^{\prime}$, and we assume that the fibers passing through the points are $F_{j}(1 \leq j \leq q)$. We blow up these $q$ points, with exceptional divisors $E_{j}^{(1)}$; then blow up the intersection points of all $E_{j}^{(1)}$ with $E^{\prime}$, with exceptional divisors $E_{j}^{(2)}$; then blow up the intersection points of all $E_{j}^{(2)}$ with $E^{\prime}$; and so forth. We do this type of blow-up $p>1$ times, where $p$ satisfies $\operatorname{gcd}(q, p)=1$ :

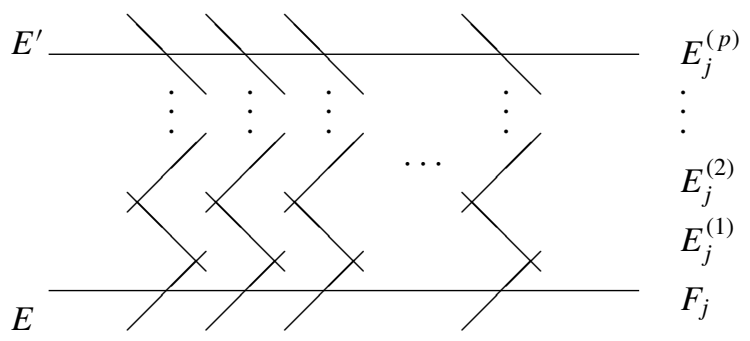

Now we contract the curves $E, E^{\prime}$, and $E_{j}^{(1)}, E_{j}^{(2)}, \ldots, E_{j}^{(p-1)}$ and thereby obtain the sought-for $\log$ del Pezzo surface $S$ that satisfies $\operatorname{Pic}(S)^{G}=\mathbb{Z}$. In fact, $e$ is the only element of $G$ that fixes $E_{j}^{(i)}$ pointwise, so $G$ acts on $S$ with IFP. By [Mu], we know that $\pi_{1}\left(S^{\mathrm{sm}}\right)$ is the finite group generated by $a, b, c_{j}(1 \leq j \leq q)$ with the relations $a^{p}=1, b^{p q-q}=1,\left(c_{j}\right)^{p}=1, a=c_{j}^{-1}$, and $b=c_{j}$. $\operatorname{But} \operatorname{gcd}(p, q)=$ 1 implies that this group is actually trivial.

5.3. REMARK. The remaining cases are when $S=\mathbb{P}^{1} \times \mathbb{P}^{1}$ and $G$ is one of the following groups: $\left(D_{2 m}, \mathbb{Z} / m, O, T\right)(\operatorname{gcd}(m, 6)=1),\left(D_{6 m}, \mathbb{Z} / m, O,(\mathbb{Z} / 2)^{2}\right)$ $(\operatorname{gcd}(m, 2)=1)$, or $\left(D_{2 m}, \mathbb{Z} / m, D_{4 n}, D_{2 n}\right)(\operatorname{gcd}(m, 2 n)=1)$. Arguing as before, we show that these groups can act on a del Pezzo surface $S$ with IFP and that $\pi_{1}\left(S^{\mathrm{sm}}\right)=\mathbb{Z} / 2$. The best we can do with these cases is as follows.

5.4. Question. Let $S$ be any (rank-1) log del Pezzo surface on which a finite group $G$ acts such that the action $(S, G)$ is with IFP and is also birational to one of the actions listed in Remark 5.3. Then does $\pi_{1}\left(S^{\mathrm{sm}}\right)$ always contain a subgroup that is isomorphic to $\mathbb{Z} / 2$ ?

\section{References}

[A] V. Alexeev, Two two-dimensional terminations, Duke Math. J. 69 (1993), 527-545.

[B] H. F. Blichfeldt, Finite collineation groups, with an introduction to the theory of groups of operators and substitution groups, Univ. Chicago Press, Chicago, 1917.

[Br] E. Brieskorn, Rationale Singularitäten komplexer Flächen, Invent. Math. 4 (1967/1968), 336-358.

[D] I. Dolgachev, Topics in classical algebraic geometry, Part I, Lecture notes, 2006, $\langle$ www.math.las.umich.edu/idolga/lecturenotes.html $\rangle$.

[DI] I. Dolgachev and V. Iskovskikh, Finite subgroups of plane cremona group, Algebra, arithmetic and geometry-Manin Festschriftmath, 2006, AG/0610595 v3.

[SGA1] A. Grothendieck, Revêtements étales et groupe fondamental, Lecture Notes in Math., 224, Springer-Verlag, Berlin, 1971. 
[GZ] R. V. Gurjar and D.-Q. Zhang, $\pi_{1}$ of smooth points of a log del Pezzo surface is finite. I, II. J. Math. Sci. Univ. Tokyo 1 (1994), 137-180; 2 (1995), 165-196.

[KMc] S. Keel and J. McKernan, Rational curves on quasi-projective surfaces, Mem. Amer. Math. Soc. 140 (1999).

[Ko] J. Kollár, Positive Sasakian structures on 5-manifolds, preprint, 2006, math.AG/0612524.

[KoM] J. Kollár and S. Mori, Birational geometry of algebraic varieties, Cambridge Tracts in Math., 134, Cambridge Univ. Press, Cambridge, 1998.

$[\mathrm{Mu}] \mathrm{D}$. Mumford, The topology of normal singularities of an algebraic surface and a criterion for simplicity, Inst. Hautes Études Sci. Publ. Math. 9 (1961), 5-22.

Department of Mathematics

Fine Hall, Washington Road

Princeton, NJ 08540

chenyang@math.princeton.edu

Current address

Department of Mathematics

Massachusetts Institute of Technology

77 Massachusetts Avenue

Cambridge, MA 02139

cyxu@mit.edu 